\title{
Free-Form Laminated Doubly-Curved Shells and Panels of Revolution Resting on Winkler-Pasternak Elastic Foundations: A 2-D GDQ Solution for Static and Free Vibration Analysis
}

\author{
Francesco Tornabene $^{1 *}$, Alessandro Ceruti ${ }^{2}$ \\ ${ }^{1}$ DICAM Department, School of Engineering, University of Bologna, Bologna, Italy \\ ${ }^{2}$ DIN Department, School of Engineering, University of Bologna, Bologna, Italy \\ Email: *francesco.tornabene@unibo.it, alessandro.ceruti@unibo.it
}

Received November 18, 2012; revised December 19, 2012; accepted December 28, 2012

\begin{abstract}
This work presents the static and dynamic analyses of laminated doubly-curved shells and panels of revolution resting on Winkler-Pasternak elastic foundations using the Generalized Differential Quadrature (GDQ) method. The analyses are worked out considering the First-order Shear Deformation Theory (FSDT) for the above mentioned moderately thick structural elements. The effect of the shell curvatures is included from the beginning of the theory formulation in the kinematic model. The solutions are given in terms of generalized displacement components of points lying on the middle surface of the shell. Simple Rational Bézier curves are used to define the meridian curve of the revolution structures. The discretization of the system by means of the GDQ technique leads to a standard linear problem for the static analysis and to a standard linear eigenvalue problem for the dynamic analysis. Comparisons between the present formulation and the Reissner-Mindlin theory are presented. Furthermore, GDQ results are compared with those obtained by using commercial programs. Very good agreement is observed. Finally, new results are presented in order to investigate the effects of the Winkler modulus, the Pasternak modulus and the inertia of the elastic foundation on the behavior of laminated shells of revolution.
\end{abstract}

Keywords: Doubly-Curved Shells of Revolution; Rational Bézier Curves; Laminated Composite Shells; Winkler-Pasternak Foundation; First-Order Shear Deformation Theory; Generalized Differential Quadrature Method

\section{Introduction}

During the last sixty years, two-dimensional linear theories of thin shells have been developed including important contributions by Timoshenko and Woinowsky-Krieger [1], Flügge [2], Gol'denveizer [3], Novozhilov [4], Vlasov [5], Ambartusumyan [6], Kraus [7], Leissa [8,9], Markuš [10], Ventsel and Krauthammer [11] and Soedel [12]. All these contributions are based on the KirchhoffLove assumptions. The transverse shear deformation has been incorporated into shell theories by following the theory of Reissner-Mindlin [13], also named First-order Shear Deformation Theory (FSDT). Abandoning the assumption related to the preservation of the normals to the shell middle surface after the deformation, a comprehensive analysis for elastic isotropic shells was made by Kraus [7], Gould [14,15] and Qatu [16,17]. The present work is just based on the FSDT. In order to include the

*Corresponding author. effect of the initial curvature in the evaluation of the stress resultants a generalization of the Reissner-Mindlin (RM) theory has been proposed in literature by Kraus [7], Qatu [16,17] and Toorani and Lakis [18,19]. There are three different ways to evaluate the engineering elastic constants in the study of curved shells. The first is the Reissner-Mindlin approach [7] that consists in neglecting the effect of curvatures. Using this approach the engineering elastic stiffnesses are constant and do not depend on curvatures. The second one, proposed by Kraus [7] and Toorani and Lakis [18], is based on the Taylor expansion, while the third one proposed by Qatu [16] consists in the exact integration of the elastic constants. As a consequence of the use of these considerations, the stress resultants directly depend on the geometry of the structure in terms of the curvature coefficients. In this latter case, the hypothesis of the symmetry of the in-plane shearing force resultants and the torsional couples de- 
clines. A further improvement of the previous theories of shells has been proposed by Toorani and Lakis [19]. In the present work their kinematic model is used in order to include the effect of the curvature from the beginning of the shell formulation. In this way, the strain relationships have to change and, as a consequence, the equilibrium equations in terms of displacements have to be modified. In the present paper, the proposed shell theory, named General Shell Theory (GST), is considered and compared with the Reissner-Mindlin (RM) theory. Comparisons between these two different formulations are presented in this paper. Several studies dealing with the shells theory have been presented years before. The most popular numerical tool used to perform the static and dynamic analyses is currently the finite element method $[14,15,20]$. The generalized collocation method based on the ring element method has also been applied. In this method, each static and kinematic variable is transformed into a theoretically infinite Fourier series of harmonic components, with respect to the circumferential co-ordinate [21,22]. In other words, when dealing with a completely closed shell, the 2D problem can be reduced using standard Fourier decomposition. For a panel, however, it is not possible to perform such a reduction operation, and the two dimensional field must be directly dealt, as it will just be done in the present work. Furthermore, the system of second-order linear partial differential equations is solved, without resorting to the one-dimensional formulation of the equilibrium of the shell. Complete revolution shells are obtained as special cases of shell panels by satisfying the kinematical and physical compatibility at the common meridian with $\vartheta=0,2 \pi$. The excellent mathematical and computational algorithmic properties, combined with successful industrial applications, have contributed to the enormous popularity of the Rational Bézier and Non-Uniform Rational B-Splines (NURBS) curves [23-25]. These curves allow to generalize the shape of the shell meridian and can be used for the optimization of the structure itself. By introducing the Differential Quadrature rule [26] and the simple mathematical formulation of the Rational Bézier and NURBS curves [23-25], it is possible to numerically evaluate the geometric parameters of a free-form shell of revolution. For the sake of simplicity and without loosing generality, only Rational Bézier curves are used in this study. Due to the increasing importance of the interaction of shells with the elastic medium, the Winkler-Pasternak foundation is introduced. Differently from papers presented in literature [27-31], all the effects of the foundation, except the damping, are separately considered. New results are presented in order to investigate the effects of the Winkler modulus, the Pasternak modulus and the inertia of the elastic foundation on the behavior of laminated shells of revolution. The mathematical fundamen- tals and recent developments of the GDQ method as well as its major applications in engineering are discussed in detail in the book by Shu [26]. The interest of researches in this procedure is increasing due to its great simplicity and versatility. As shown in the literature [32], GDQ technique is a global method which can obtain very accurate numerical results by using a considerably small number of grid points. Therefore, this simple direct procedure has been applied in a large number of cases [33$85]$ to circumvent the difficulties of programming complex algorithms for the computer, as well as to reduce the computational time. In conclusion, the aim of the present paper is to demonstrate an efficient and accurate application of the Differential Quadrature approach, by solving the equations governing the static and the free vibration of laminated composite doubly-curved moderately thick shells and panels of revolution. Summarizing, this research deals with four aspects. The first is the improvement of the Reissner-Mindlin Theory using a different kinematical model. In this way the effect of the curvature of the shell structure is considered from the beginning of the theory derivation. The second is the generalization of the shape of the shell meridian. The Differential Quadrature rule is used to evaluate the geometric parameters needed to describe the geometry of the structure when a Rational Bézier meridian curve is assumed. The third is the investigation of the effects of Winkler-Pasternak foundations on the behavior of the shell structures in static and dynamic analyses. All the effects of the foundation are separately considered. The fourth is the use of the Generalized Differential Quadrature method to solve the governing shell equations.

\section{Shell Fundamental Equations}

The basic configuration of the problem herein considered is a laminated composite doubly-curved shell [83] as shown in Figure 1. The co-ordinates along the meridian and circumferential directions of the reference surface are $\varphi$ and $s$, respectively. The distance of each point from the shell mid-surface along the normal is $\zeta$. It is considered a laminated composite shell made of $l$ laminae or plies, where the total thickness of the shell $h$ is defined as:

$$
h=\sum_{k=1}^{l} h_{k}
$$

in which $h_{k}=\zeta_{k+1}-\zeta_{k}$ is the thickness of the $k$-th lamina or ply. In this work, doubly-curved shells of revolution are considered. For this type of structures the analytical expressions of the meridian curve are reported in the work by Tornabene [78], so that no further considerations will be introduced. The angle formed by the extended normal $\boldsymbol{n}$ to the reference surface and the axis of rotation $x_{3}$, or the geometric axis $x_{3}^{\prime}$ of the meridian 
curve, is defined as the meridian angle $\varphi$; the angle between the radius of the parallel circle $R_{0}(\varphi)$ and the $x_{1}$ axis is designated as the circumferential angle $\vartheta$, as shown in Figure 2.

For these structures the parametric co-ordinates $(\varphi, s)$ define, respectively, the meridian curves and the parallel circles upon the middle surface of the shell. The curvilinear abscissa $s(\varphi)$ of a generic parallel is related to the circumferential angle $\vartheta$ by the relation $s=\vartheta R_{0}$. The horizontal radius $R_{0}(\varphi)$ of a generic parallel of the shell represents the distance of each point from the axis of revolution $x_{3} . R_{b}$ is the shift of the geometric axis of the curved meridian $x_{3}^{\prime}$ with reference to the axis of revolution $x_{3}$. The position of an arbitrary point within the shell material is defined by co-ordinates

$\varphi\left(\varphi_{0} \leq \varphi \leq \varphi_{1}\right), \quad s\left(0 \leq s \leq s_{0}\right)$ upon the middle surface, and $\zeta$ directed along the outward normal and measured from the reference surface $(-h / 2 \leq \zeta \leq h / 2)$. The geometry of shells considered [83] is a surface of revolution (Figure 2).

A simple way to define a general meridian curve is to use the well-known Rational Bézier representation of a plane curve $[24,25,80]$. In particular, it is possible to describe a Rational Bézier curve in the following manner:

$$
\begin{aligned}
& \hat{x}_{1}(u)=\sum_{i=0}^{n} B_{i, n}(u) w_{i} \bar{x}_{1 i} / \sum_{i=0}^{n} B_{i, n}(u) w_{i} \\
& \hat{x}_{3}^{\prime}(u)=\sum_{i=0}^{n} B_{i, n}(u) w_{i} \bar{x}_{3 i}^{\prime} / \sum_{i=0}^{n} B_{i, n}(u) w_{i}
\end{aligned}
$$

where $u \in[0,1]$ is the curve parameter, $w_{i}$ are the weight coefficients and $\left(\bar{x}_{1 i}, \bar{x}_{3 i}^{\prime}\right)$ are the co-ordinates of the curve control points. Furthermore, the classical $n$-th degree Bernstein polynomial formulations are given by:

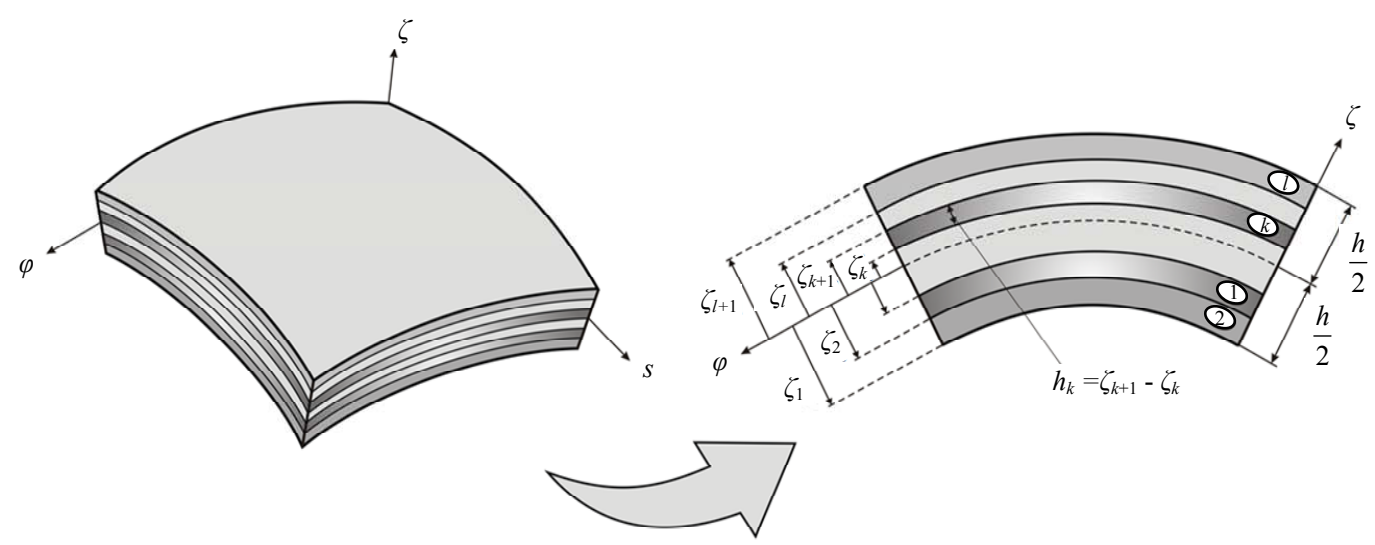

Figure 1. Co-ordinate system of a laminated composite doubly-curved shell.
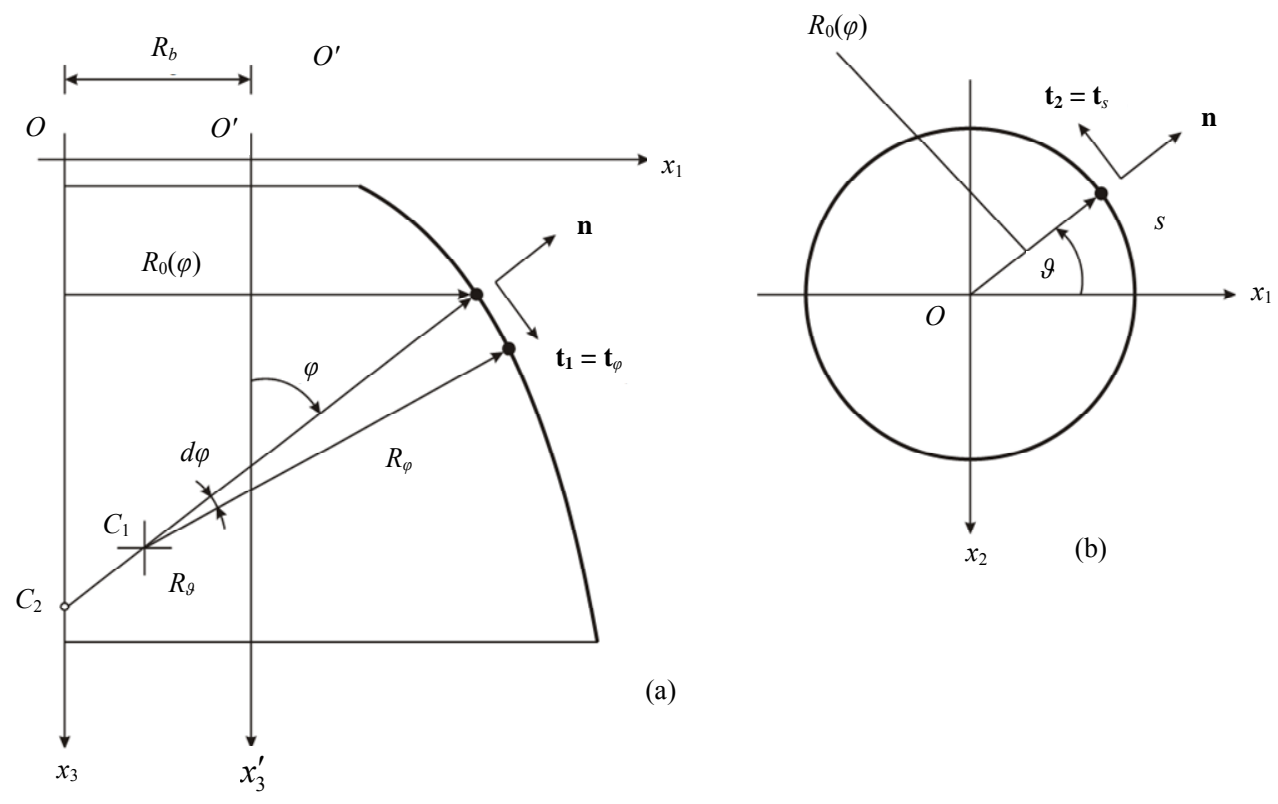

Figure 2. Shell geometry: Meridian section (a); Circumferential section (b). 


$$
B_{i, n}(u)=\frac{n !}{i !(n-i) !} u^{i}(1-u)^{n-i}
$$

In this way, only the co-ordinates of the curve $\left(\hat{x}_{1 i}, \hat{x}_{3 i}^{\prime}\right), i=1,2, \cdots, m$, are known in the co-ordinate reference system $O^{\prime} x_{1} x_{3}^{\prime}$. In order to solve the shell problem, it is important to express the horizontal radius $R_{0}(\varphi)$ of a generic parallel and the radii of curvature $R_{\varphi}(\varphi), R_{s}(\varphi)$ in the meridian and circumferential directions as functions of $\varphi$. Based on the differential geometry $[7,12,57,80,83]$, the radius of curvature of the meridian curve can be described as a function of $x_{3}^{\prime}$ using the following expression:

$$
R_{\varphi}\left(x_{3}^{\prime}\right)=\left(1+\left(\frac{\mathrm{d} x_{1}}{\mathrm{~d} x_{3}^{\prime}}\right)^{2}\right)^{\frac{3}{2}} /\left|\frac{\mathrm{d}^{2} x_{1}}{\mathrm{~d} x_{3}^{\prime 2}}\right|
$$

It is worth noting that the derivatives of the meridian curve are not known a priori, so that a numeric method to evaluate the first and second derivatives of the meridian curve is required. The differential quadrature rule allows to approximate these derivatives using the following definition [26]:

$$
\left.\frac{\mathrm{d}^{n} f(x)}{\mathrm{d} x^{n}}\right|_{x=x_{i}}=\sum_{j=1}^{N} \varsigma_{i j}^{(n)} f\left(x_{j}\right), \text { for } i=1,2, \cdots, N
$$

where $\varsigma_{i j}^{(n)}$ are the weighting coefficients of the $n$-th order derivative. By discretizing the domain

$$
I \in\left[\hat{x}_{31}^{\prime}, \hat{x}_{3 m}^{\prime}\right]
$$

using the Chebyshev-Gauss-Lobatto (C-G-L) grid distribution:

$$
\begin{aligned}
& \hat{x}_{3 i}^{\prime}=\left(1-\cos \left(\frac{i-1}{N-1} \pi\right)\right) \frac{\left(\hat{x}_{3 m}^{\prime}-\hat{x}_{31}^{\prime}\right)}{2}+\hat{x}_{31}^{\prime}, \\
& \text { for } i=1,2, \cdots, N \text {, for } \hat{x}_{3}^{\prime} \in\left[\hat{x}_{31}^{\prime}, \widehat{x}_{3 m}^{\prime}\right]
\end{aligned}
$$

and interpolating the $\hat{x}_{1}$ co-ordinates of the curve points derived by the Equations (2) using the previous calculated points (6), the general curve can be represented by the new co-ordinates points $\left(\hat{x}_{1 i}, \hat{x}_{3 i}^{\prime}\right)$, for $i=1,2, \cdots, N$. Applying the differential quadrature definition (5), the expression (4) assumes the following discrete aspect:

$$
\begin{aligned}
& R_{\varphi}\left(\hat{x}_{3 i}^{\prime}\right)=\left(1+\left(\sum_{j=1}^{N} \varsigma_{i j}^{\hat{x}_{3}^{\prime}(1)} \hat{x}_{1 i}\right)^{2}\right)^{\frac{3}{2}} /\left|\sum_{j=1}^{N} \varsigma_{i j}^{\hat{x}_{3}^{\prime}(2)} \hat{x}_{1 i}\right|, \\
& \text { for } i=1,2, \cdots, N
\end{aligned}
$$

where $\varsigma_{i j}^{\hat{x}_{3}^{\prime}(n)}$ are the weighting coefficients evaluated in the domain $I \in\left[\widehat{x}_{31}^{\prime}, \hat{x}_{3 m}^{\prime}\right]$. As a results of the differential geometry $[7,12,57,80,83]$, it is possible to introduce the following relation:

$$
\varphi=\frac{\pi}{2}-\arctan \left(\frac{\mathrm{d} x_{1}}{\mathrm{~d} x_{3}^{\prime}}\right)
$$

By using the differential quadrature definition (5), the relation (7) can be expressed in the discrete form:

$$
\begin{aligned}
& \hat{\varphi}_{i}=\hat{\varphi}\left(\hat{x}_{3 i}^{\prime}\right)=\frac{\pi}{2}-\arctan \left(\sum_{j=1}^{N} \varsigma_{i j}^{\hat{x}_{3}^{\prime}(1)} \hat{x}_{1 i}\right), \\
& \text { for } i=1,2, \cdots, N
\end{aligned}
$$

By discretizing the domain $I_{\varphi} \in\left[\hat{\varphi}_{1}, \hat{\varphi}_{N}\right]$ using the Chebyshev-Gauss-Lobatto (C-G-L) grid distribution:

$$
\begin{aligned}
& \varphi_{i}=\left(1-\cos \left(\frac{i-1}{N-1} \pi\right)\right) \frac{\left(\hat{\varphi}_{N}-\hat{\varphi}_{1}\right)}{2}+\hat{\varphi}_{1}, \\
& \text { for } i=1,2, \cdots, N, \text { for } \varphi \in\left[\hat{\varphi}_{1}, \hat{\varphi}_{N}\right]
\end{aligned}
$$

and interpolating the $\hat{x}_{1}$ and $\hat{x}_{3}^{\prime}$ co-ordinates of the curve points using the calculated points (9), the general curve can be represented by the following new co-ordinate points $\left(\tilde{x}_{1 i}, \tilde{x}_{3 i}^{\prime}\right)$, for $i=1,2, \cdots, N$. Thus, all the discrete points of the curve are determined in terms of the co-ordinates $\left(\tilde{x}_{1 i}, \tilde{x}_{3 i}^{\prime}\right)$ and the angle $\varphi_{i}$. In the Figure 3, a Rational Bézier curve, its control points and the curve co-ordinates $\left(\tilde{x}_{1 i}, \tilde{x}_{3 i}^{\prime}\right)$, evaluated as above exposed, are represented [80]. The vectors of the control points and the weights used in Figure 3 are the following:

$$
\begin{aligned}
& \overline{\boldsymbol{x}}_{1}=\left[\begin{array}{llllll}
0.2 & 0.7 & 1.2 & 1.4 & 1.4 & 1.2
\end{array}\right] \\
& \overline{\boldsymbol{x}}_{3}^{\prime}=\left[\begin{array}{llllll}
0 & 0.2 & 0.6 & 1 & 1.5 & 2
\end{array}\right] \\
& \boldsymbol{w}=\left[\begin{array}{llllll}
1 & 1 & 1 & 1 & 1 & 1
\end{array}\right]
\end{aligned}
$$

Based on the previous considerations, the horizontal radius $R_{0}(\varphi)$ of a shell of revolution assumes the following discrete form:

$$
R_{0 i}=R_{0}\left(\varphi_{i}\right)=\tilde{x}_{1 i}+R_{b}, \text { for } i=1,2, \cdots, N
$$

For doubly-curved revolution shells the Gauss-Codazzi relation can be expressed as follows:

$$
\frac{\mathrm{d} R_{0}}{\mathrm{~d} \varphi}=R_{\varphi} \cos \varphi
$$

By using the differential quadrature definition (5), it is possible to determine the radius of curvature $R_{\varphi}(\varphi)$ in meridian direction and its first and second derivatives in discrete form:

$$
\begin{aligned}
& R_{\varphi i}=R_{\varphi}\left(\varphi_{i}\right)=\frac{1}{\cos \varphi_{i}} \sum_{j=1}^{N} \varsigma_{i j}^{\varphi(1)} R_{0 i}, \text { for } i=1,2, \cdots, N \\
& \left.\frac{\mathrm{d} R_{\varphi}}{\mathrm{d} \varphi}\right|_{i}=\left.\frac{\mathrm{d} R_{\varphi}}{\mathrm{d} \varphi}\right|_{\varphi_{i}}=\sum_{j=1}^{N} \varsigma_{i j}^{\varphi(1)} R_{\varphi i}, \text { for } i=1,2, \cdots, N \\
& \left.\frac{\mathrm{d}^{2} R_{\varphi}}{\mathrm{d} \varphi^{2}}\right|_{i}=\left.\frac{\mathrm{d}^{2} R_{\varphi}}{\mathrm{d} \varphi^{2}}\right|_{\varphi_{i}}=\sum_{j=1}^{N} \varsigma_{i j}^{\varphi(2)} R_{\varphi i}, \text { for } i=1,2, \cdots, N
\end{aligned}
$$




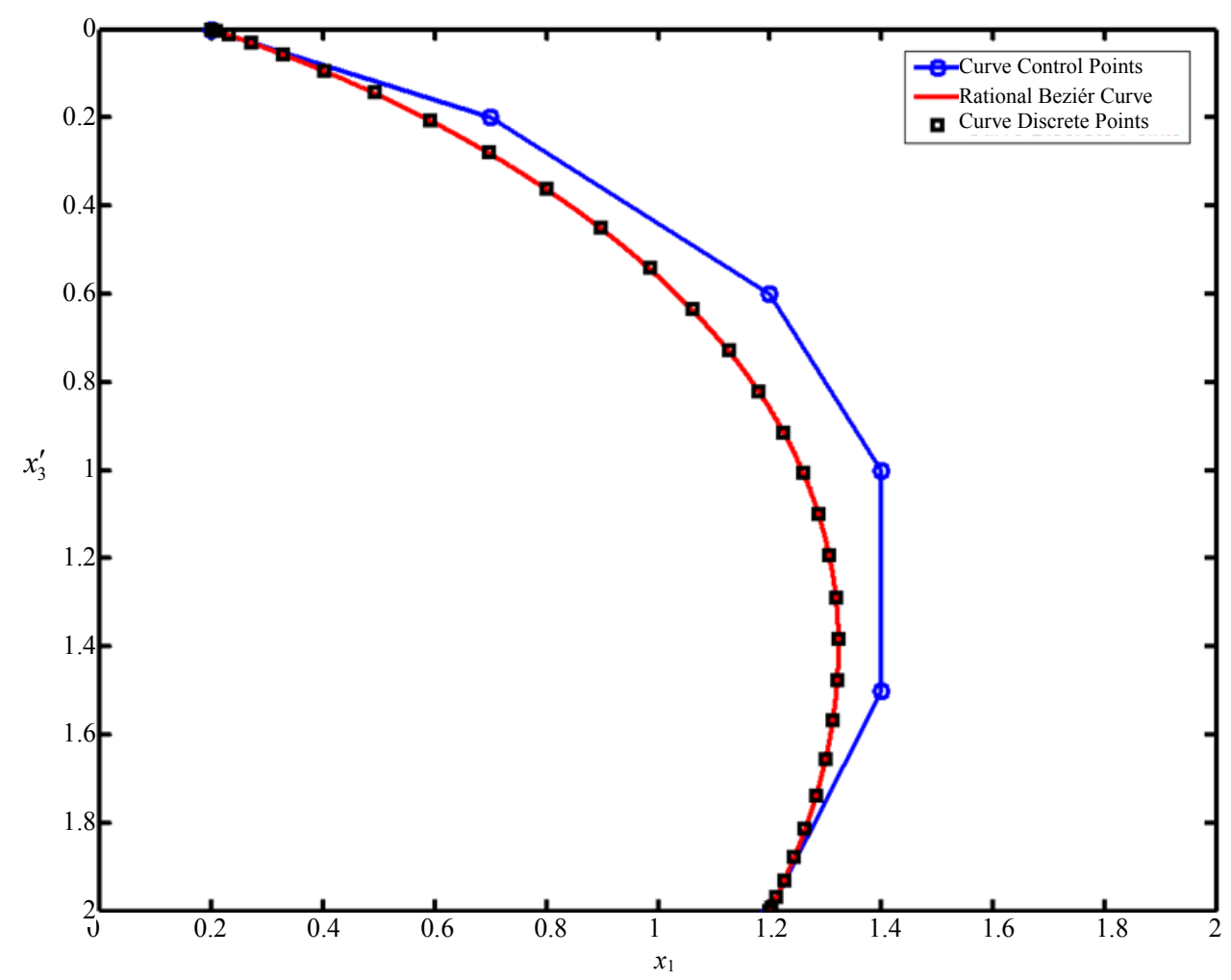

Figure 3. A Rational Bézier curve $\left(\hat{x}_{1 i}, \hat{x}_{3 i}^{\prime}\right)$, its control points $\left(\hat{x}_{1 i}, \hat{x}_{3 i}^{\prime}\right)$ and curve evaluated discrete points $\left(\hat{x}_{1 i}, \hat{x}_{3 i}^{\prime}\right)$.

Finally, as a results of the differential geometry $[7,12$, $57,80,83]$, the radius of curvature $R_{s}(\varphi)$ in circumferential direction for a shell of revolution can be expressed in a discrete form as follows:

$$
R_{s i}=R_{s}\left(\varphi_{i}\right)=\frac{R_{0 i}}{\sin \varphi_{i}}, \text { for } i=1,2, \cdots, N
$$

It is worth noting that, following the previous considerations, all the useful geometric parameters describing the surface of revolution under consideration are known in discrete form (11)-(16). As shown, the differential quadrature rule (5) has been used to approximate the derivatives needed for the definition of the geometry of a shell of revolution. As concerns the shell theory, the present work is based on the following assumptions: 1) the transverse normal is inextensible so that the normal strain is equal to zero: $\left.\varepsilon_{n}=\varepsilon_{n}(\varphi, s, \zeta, t)=0 ; 2\right)$ the transverse shear deformation is considered to influence the governing equations so that normal lines to the reference surface of the shell before deformation remain straight, but not necessarily normal after deformation (a relaxed KirchhoffLove hypothesis); 3) the shell deflections are small and the strains are infinitesimal; 4) the shell is moderately thick, therefore it is possible to assume that the thickness direction normal stress is negligible so that the in-plane assumption can be invoked: $\left.\sigma_{n}=\sigma_{n}(\varphi, s, \zeta, t)=0 ; 5\right)$ the linear elastic behavior of anisotropic materials is as- sumed; 6) the rotary inertia and the initial curvature are also taken into account. Consistent with the assumptions of a moderately thick shell theory reported above, the displacement field considered in this study follows the First-order Shear Deformation Theory and it can be put in the following form:

$$
\begin{aligned}
& U_{\varphi}(\varphi, s, \zeta, t)=\left(1+\frac{\zeta}{R_{\varphi}}\right) u_{\varphi}(\varphi, s, t)+\zeta \beta_{\varphi}(\varphi, s, t) \\
& U_{s}(\varphi, s, \zeta, t)=\left(1+\frac{\zeta}{R_{s}}\right) u_{s}(\varphi, s, t)+\zeta \beta_{s}(\varphi, s, t) \\
& W(\varphi, s, \zeta, t)=w(\varphi, s, t)
\end{aligned}
$$

where $u_{\varphi}, u_{s}, w$ are the displacement components of points lying on the middle surface $(\zeta=0)$ of the shell, along meridian, circumferential and normal directions, respectively, while $t$ is the time variable. $\beta_{\varphi}$ and $\beta_{s}$ are normal-to-mid-surface rotations, respectively. The kinematic hypothesis expressed by Equations (17) should be supplemented by the statement that the shell deflections are small and strains are infinitesimal, that is $w(\varphi, s, t) \ll h$. The in-plane displacements $U_{\varphi}$ and $U_{s}$ vary linearly through the thickness, while $\stackrel{\varphi}{W}$ remains independent of $\zeta$. It should be also remarked that, differently from the previous works by Tornabene $[65,77,78]$, the displacement field has been improved taking into 
account the real geometry of the shell and in particular the curvature effect has been directly introduced into the kinematical model as proposed by Toorani and Lakis [19].
Due to the change of the kinematical model the relationships between strains and displacements along the shell reference surface $(\zeta=0)$ become the following:

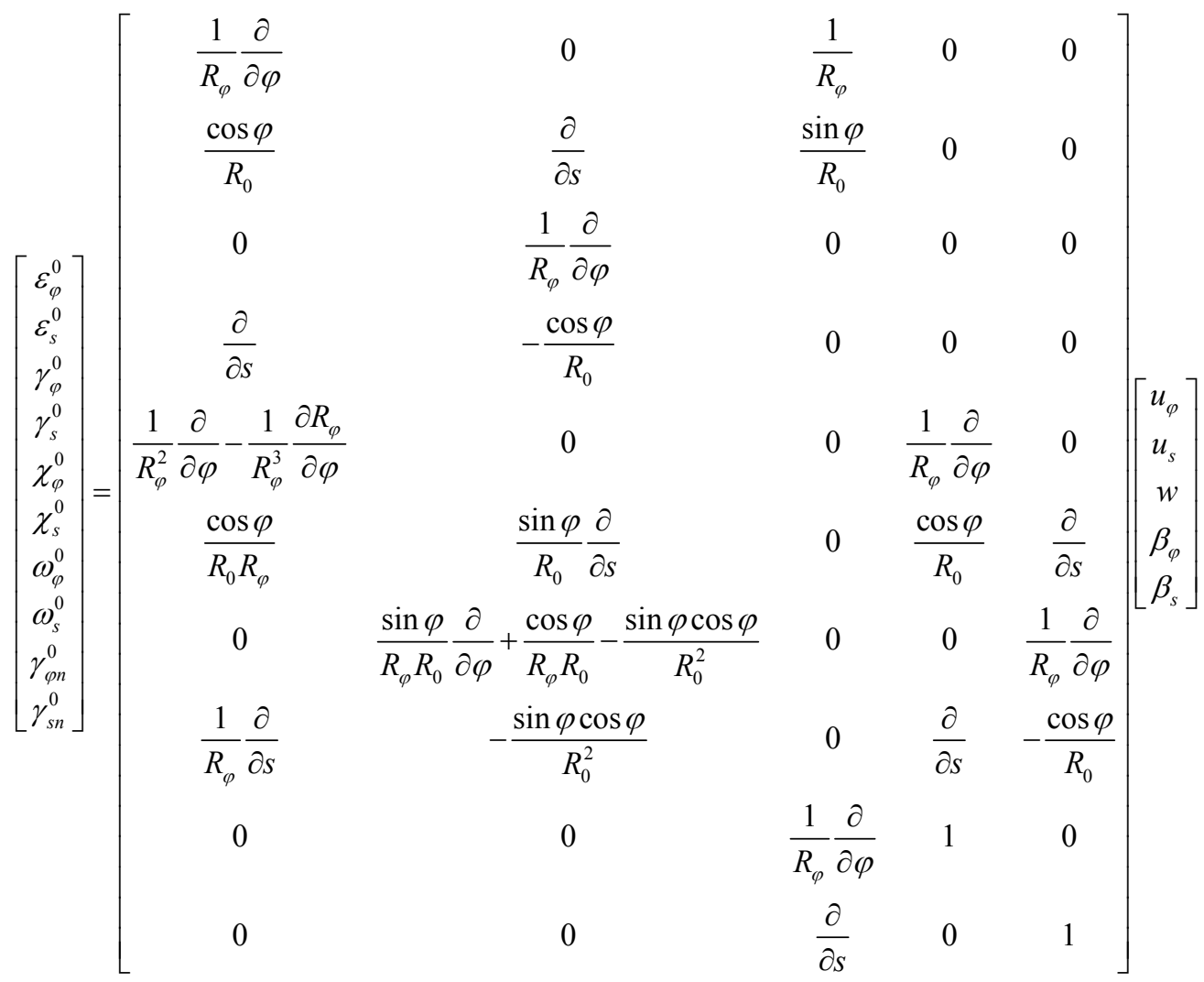

that are different from those presented in previous papers $[77,78]$. In the above Equations (19), the first four strains $\varepsilon_{\varphi}^{0}, \varepsilon_{s}^{0}, \gamma_{\varphi}^{0}, \gamma_{s}^{0}$ are the in-plane meridian, circumferential and shearing components, and $\chi_{\varphi}^{0}, \chi_{s}^{0}, \omega_{\varphi}^{0}, \omega_{s}^{0}$ are the analogous curvature changes. The last two components $\gamma_{\varphi n}^{0}, \gamma_{s n}^{0}$ are the transverse shearing strains. The shell composition assumed in the following is a laminated composite linear elastic material. Accordingly, the following constitutive equations relate internal stress resultants and internal couples with generalized strain components (18) on the middle surface:

$$
\left[\begin{array}{c}
N_{\varphi} \\
N_{s} \\
N_{\varphi s} \\
N_{s \varphi} \\
M_{\varphi} \\
M_{s} \\
M_{\varphi s} \\
M_{s \varphi} \\
T_{\varphi} \\
T_{s}
\end{array}\right]=\left[\begin{array}{cccccccccc}
\bar{A}_{11 \varphi}^{(0)} & A_{12}^{(0)} & \bar{A}_{16 \varphi}^{(0)} & A_{16}^{(0)} & \bar{A}_{11 \varphi}^{(1)} & A_{12}^{(1)} & \bar{A}_{16 \varphi}^{(1)} & A_{16}^{(1)} & 0 & 0 \\
A_{12}^{(0)} & \bar{A}_{22 s}^{(0)} & A_{26}^{(0)} & \bar{A}_{26 s}^{(0)} & A_{12}^{(1)} & \bar{A}_{22 s}^{(1)} & A_{26}^{(1)} & \bar{A}_{26 s}^{(1)} & 0 & 0 \\
\bar{A}_{16 \varphi}^{(0)} & A_{26}^{(0)} & \bar{A}_{66 \varphi}^{(0)} & A_{66}^{(0)} & \bar{A}_{16 \varphi}^{(1)} & A_{26}^{(1)} & \bar{A}_{66 \varphi}^{(1)} & A_{66}^{(1)} & 0 & 0 \\
A_{16}^{(0)} & \bar{A}_{26 s}^{(0)} & A_{66}^{(0)} & \bar{A}_{66 s}^{(0)} & A_{16}^{(1)} & \bar{A}_{26 s}^{(1)} & A_{66}^{(1)} & \bar{A}_{66 s}^{(1)} & 0 & 0 \\
\bar{A}_{11 \varphi}^{(1)} & A_{12}^{(1)} & \bar{A}_{16 \varphi}^{(1)} & A_{16}^{(1)} & \bar{A}_{11 \varphi}^{(2)} & A_{12}^{(2)} & \bar{A}_{16 \varphi}^{(2)} & A_{16}^{(2)} & 0 & 0 \\
A_{12}^{(1)} & \bar{A}_{22 s}^{(1)} & A_{26}^{(1)} & \bar{A}_{26 s}^{(1)} & A_{12}^{(2)} & \bar{A}_{22 s}^{(2)} & A_{26}^{(2)} & \bar{A}_{26 s}^{(2)} & 0 & 0 \\
\bar{A}_{16 \varphi}^{(1)} & A_{26}^{(1)} & \bar{A}_{66 \varphi}^{(1)} & A_{66}^{(1)} & \bar{A}_{16 \varphi}^{(2)} & A_{26}^{(2)} & \bar{A}_{66 \varphi}^{(2)} & A_{66}^{(2)} & 0 & 0 \\
A_{16}^{(1)} & \bar{A}_{26 s}^{(1)} & A_{66}^{(1)} & \bar{A}_{66 s}^{(1)} & A_{16}^{(2)} & \bar{A}_{26 s}^{(2)} & A_{66}^{(2)} & \bar{A}_{66 s}^{(2)} & 0 & 0 \\
0 & 0 & 0 & 0 & 0 & 0 & 0 & 0 & \kappa \bar{A}_{44 \varphi}^{(0)} & \kappa A_{45}^{(0)} \\
0 & 0 & 0 & 0 & 0 & 0 & 0 & 0 & \kappa A_{45}^{(0)} & \kappa \bar{A}_{55 s}^{(0)}
\end{array}\right]\left[\begin{array}{c}
\varepsilon_{\varphi}^{0} \\
\varepsilon_{s}^{0} \\
\gamma_{\varphi}^{0} \\
\gamma_{s}^{0} \\
\chi_{\varphi}^{0} \\
\chi_{s}^{0} \\
\omega_{\varphi}^{0} \\
\omega_{s}^{0} \\
\gamma_{\varphi n}^{0} \\
\gamma_{s n}^{0}
\end{array}\right]
$$

where the elastic engineering stiffnesses $\bar{A}_{i j m}^{(q)}$ which depend on curvatures are defined as follows (see Appendix for 
more details):

$$
\begin{aligned}
& \bar{A}_{i j m}^{(q)} \cong A_{i j}^{(q)}+\left(\frac{1}{R_{s}}-\frac{1}{R_{\varphi}}\right) \sum_{p=1}^{r}(-1)^{p+n_{m}} \frac{A_{i j}^{(q+p)}}{R_{m}^{p-1}}=\sum_{k=1}^{l}\left(\int_{\zeta_{k}}^{\zeta_{k+1}} \bar{Q}_{i j}^{(k)} \zeta^{q} \mathrm{~d} \zeta+\left(\frac{1}{R_{s}}-\frac{1}{R_{\varphi}}\right) \sum_{p=1}^{r} \frac{(-1)^{p+n_{m}}}{R_{m}^{p-1}} \int_{\zeta_{k}}^{\zeta_{k+1}} \bar{Q}_{i j}^{(k)} \zeta^{q+p} \mathrm{~d} \zeta\right) \\
& m=\varphi, s, n_{\varphi}=1, n_{s}=2, q=0,1,2, r=1,2,3, \cdots, 20
\end{aligned}
$$

Several approaches can be found in literature to evaluate the engineering elastic constants $\bar{A}_{i j m}^{(q)} \quad[7,16-18]$. It is worth noting that due to the fact that the elastic engineering stiffnesses $\bar{A}_{i j m}^{(q)}$ depend on curvatures, the corresponding derivatives respect to the co-ordinates along the meridian $\varphi$ and circumferential $s$ directions of the reference surface have to be evaluated. In order to perform this operation, the Differential Quadrature rule [26] is used. Thus, the derivatives of the elastic engineering stiffnesses $\bar{A}_{i j m}^{(q)}$ are numerically evaluated. $\kappa$ is the shear correction factor, which is usually taken equal to $\kappa=5 / 6$, such as in the present work. In particular, the determination of shear correction factors for composite laminated structures is still an unresolved issue, because these factors depend on various parameters [18]. In Equa- tions (19), the four components $N_{\varphi}, N_{s}, N_{\varphi s}, N_{s \varphi}$ are the in-plane meridian, circumferential and shearing force resultants, and $M_{\varphi}, M_{s}, M_{\varphi s}, M_{s \varphi}$ are the analogous couples, while $T_{\varphi}, T_{s}$ are the transverse shear force resultants. In the above definitions (19) the symmetry of shearing force resultants $N_{\varphi s}, N_{s \varphi}$ and torsional couples $M_{\varphi s}, M_{s \varphi}$ is not assumed as a further hypothesis, as done in Reissner-Mindlin theory. This hypothesis is in fact satisfied only in the case of spherical shells and flat plates. The assumption under discussion is derived from the consideration that ratios $\zeta / R_{\varphi}, \zeta / R_{s}$ cannot be neglected with respect to unity. For the $k$-th orthotropic lamina the elastic constants $\bar{Q}_{i j}^{(k)}$ in the laminate co-ordinate system $O^{\prime} \varphi s \zeta$ can be written as:

$$
\begin{aligned}
& \bar{Q}_{11}^{(k)}=Q_{11}^{(k)} \cos ^{4} \theta^{(k)}+2\left(Q_{12}^{(k)}+2 Q_{66}^{(k)}\right) \sin ^{2} \theta^{(k)} \cos ^{2} \theta^{(k)}+Q_{22}^{(k)} \cos ^{4} \theta^{(k)} \\
& \bar{Q}_{12}^{(k)}=\left(Q_{11}^{(k)}+Q_{22}^{(k)}-4 Q_{66}^{(k)}\right) \sin ^{2} \theta^{(k)} \cos ^{2} \theta^{(k)}+Q_{12}^{(k)}\left(\sin ^{4} \theta^{(k)}+\cos ^{4} \theta^{(k)}\right) \\
& \bar{Q}_{22}^{(k)}=Q_{11}^{(k)} \sin ^{4} \theta^{(k)}+2\left(Q_{12}^{(k)}+2 Q_{66}^{(k)}\right) \sin ^{2} \theta^{(k)} \cos ^{2} \theta^{(k)}+Q_{22}^{(k)} \cos ^{4} \theta^{(k)} \\
& \bar{Q}_{16}^{(k)}=\left(Q_{11}^{(k)}-Q_{12}^{(k)}-2 Q_{66}^{(k)}\right) \sin \theta^{(k)} \cos ^{3} \theta^{(k)}+\left(Q_{12}^{(k)}-Q_{22}^{(k)}+2 Q_{66}^{(k)}\right) \sin ^{3} \theta^{(k)} \cos \theta^{(k)} \\
& \bar{Q}_{26}^{(k)}=\left(Q_{11}^{(k)}-Q_{12}^{(k)}-2 Q_{66}^{(k)}\right) \sin ^{3} \theta^{(k)} \cos \theta^{(k)}+\left(Q_{12}^{(k)}-Q_{22}^{(k)}+2 Q_{66}^{(k)}\right) \sin \theta^{(k)} \cos ^{3} \theta^{(k)} \\
& \bar{Q}_{66}^{(k)}=\left(Q_{11}^{(k)}+Q_{22}^{(k)}-2 Q_{12}^{(k)}-2 Q_{66}^{(k)}\right) \sin ^{2} \theta^{(k)} \cos ^{2} \theta^{(k)}+Q_{66}^{(k)}\left(\sin ^{4} \theta^{(k)}+\cos ^{4} \theta^{(k)}\right) \\
& \bar{Q}_{44}^{(k)}=Q_{44}^{(k)} \cos ^{2} \theta^{(k)}+Q_{55}^{(k)} \sin ^{2} \theta^{(k)} \\
& \bar{Q}_{45}^{(k)}=\left(Q_{44}^{(k)}-Q_{55}^{(k)}\right) \cos \theta^{(k)} \sin ^{(k)} \\
& \bar{Q}_{55}^{(k)}=Q_{55}^{(k)} \cos ^{2} \theta^{(k)}+Q_{44}^{(k)} \sin ^{2} \theta^{(k)}
\end{aligned}
$$

where $\theta^{(k)}$ is the orientation angle of the principal material co-ordinate system $O^{\prime} \hat{\varphi} \hat{s} \hat{\zeta}$ of the $k$-th orthotropic ply with respect to the laminate co-ordinate system
$O^{\prime} \varphi s \zeta$. Furthermore, the elastic constants $Q_{i j}^{(k)}$ in the material co-ordinate system $O^{\prime} \hat{\varphi} \hat{s} \hat{\zeta}$ are expressed as follows:

$$
Q_{11}^{(k)}=\frac{E_{1}^{(k)}}{1-v_{12}^{(k)} v_{21}^{(k)}}, Q_{22}^{(k)}=\frac{E_{2}^{(k)}}{1-v_{12}^{(k)} v_{21}^{(k)}}, Q_{12}^{(k)}=\frac{v_{12}^{(k)} E_{2}^{(k)}}{1-v_{12}^{(k)} v_{21}^{(k)}}, Q_{66}^{(k)}=G_{12}^{(k)}, Q_{44}^{(k)}=G_{13}^{(k)}, Q_{55}^{(k)}=G_{23}^{(k)}
$$

where $E_{1}, E_{2}, G_{13}, G_{23}, G_{12}, v_{12}$ are the engineering parameters of the $k$-th lamina. It should be noted that for a complete characterization of an orthotropic material, the parameters $E_{3}, v_{13}, v_{23}$ have to be taken into account, as well-known. Following the virtual work principle in dynamic version, and remembering the Gauss-Codazzi relations for the shells of revolution (12), five equations of dynamic equilibrium in terms of internal actions can be 
written for the revolution shell element:

$$
\begin{aligned}
& \frac{1}{R_{\varphi}} \frac{\partial N_{\varphi}}{\partial \varphi}+\frac{\partial N_{s \varphi}}{\partial s}+\left(N_{\varphi}-N_{s}\right) \frac{\cos \varphi}{R_{0}}+\frac{1}{R_{\varphi}}\left(\frac{1}{R_{\varphi}} \frac{\partial M_{\varphi}}{\partial \varphi}+\frac{\partial M_{s \varphi}}{\partial s}+\left(M_{\varphi}-M_{s}\right) \frac{\cos \varphi}{R_{0}}\right)+q_{\varphi}=\left(I_{0}+\frac{2 I_{1}}{R_{\varphi}}+\frac{I_{2}}{R_{\varphi}^{2}}\right) \ddot{u}_{\varphi} \\
& +\left(I_{1}+\frac{I_{2}}{R_{\varphi}}\right) \ddot{\beta}_{\varphi} \frac{1}{R_{\varphi}} \frac{\partial N_{\varphi s}}{\partial \varphi}+\frac{\partial N_{s}}{\partial s}+\left(N_{\varphi s}+N_{s \varphi}\right) \frac{\cos \varphi}{R_{0}}+\frac{\sin \varphi}{R_{0}}\left(\frac{1}{R_{\varphi}} \frac{\partial M_{\varphi s}}{\partial \varphi}+\frac{\partial M_{s}}{\partial s}+\left(M_{\varphi s}+M_{s \varphi}\right) \frac{\cos \varphi}{R_{0}}\right)+q_{s} \\
& =\left(I_{0}+\frac{2 I_{1} \sin \varphi}{R_{0}}+\frac{I_{2} \sin ^{2} \varphi}{R_{0}^{2}}\right) \ddot{u}_{s}+\left(I_{1}+\frac{I_{2} \sin \varphi}{R_{0}}\right) \ddot{\beta}_{s} \frac{1}{R_{\varphi}} \frac{\partial T_{\varphi}}{\partial \varphi}+\frac{\partial T_{s}}{\partial s}+T_{\varphi} \frac{\cos \varphi}{R_{0}}-\frac{N_{\varphi}}{R_{\varphi}}-N_{s} \frac{\sin \varphi}{R_{0}}+q_{n} \\
& =I_{0} \ddot{w} \frac{1}{R_{\varphi}} \frac{\partial M_{\varphi}}{\partial \varphi}+\frac{\partial M_{s \varphi}}{\partial s}+\left(M_{\varphi}-M_{s}\right) \frac{\cos \varphi}{R_{0}}-T_{\varphi}+m_{\varphi} \\
& =\left(I_{1}+\frac{I_{2}}{R_{\varphi}}\right) \ddot{u}_{\varphi}+I_{2} \ddot{\beta}_{\varphi} \frac{1}{R_{\varphi}} \frac{\partial M_{\varphi s}}{\partial \varphi}+\frac{\partial M_{s}}{\partial s}+\left(M_{\varphi s}+M_{s \varphi}\right) \frac{\cos \varphi}{R_{0}}-T_{s}+m_{s}=\left(I_{1}+\frac{I_{2} \sin \varphi}{R_{0}}\right) \ddot{u}_{s}+I_{2} \ddot{\beta}_{s}
\end{aligned}
$$

where:

$$
\begin{aligned}
& I_{0}=\sum_{k=1}^{l} \int_{\zeta_{k}}^{\zeta_{k+1}} \rho^{(k)}\left(1+\frac{\zeta}{R_{\varphi}}\right)\left(1+\frac{\zeta \sin \varphi}{R_{0}}\right) \mathrm{d} \zeta+\frac{1}{3} \rho_{F}^{+} h_{F}^{+}\left(1+\frac{h}{2 R_{\varphi}}\right)\left(1+\frac{h \sin \varphi}{2 R_{0}}\right)+\frac{1}{3} \rho_{F}^{-} h_{F}^{-}\left(1-\frac{h}{2 R_{\varphi}}\right)\left(1-\frac{h \sin \varphi}{2 R_{0}}\right) \\
& I_{1}=\sum_{k=1}^{l} \int_{\zeta_{k}}^{\zeta_{k+1}} \rho^{(k)} \zeta\left(1+\frac{\zeta}{R_{\varphi}}\right)\left(1+\frac{\zeta \sin \varphi}{R_{0}}\right) \mathrm{d} \zeta+\frac{1}{3} \rho_{F}^{+} h_{F}^{+} \frac{h}{2}\left(1+\frac{h}{2 R_{\varphi}}\right)\left(1+\frac{h \sin \varphi}{2 R_{0}}\right)-\frac{1}{3} \rho_{F}^{-} h_{F}^{-} \frac{h}{2}\left(1-\frac{h}{2 R_{\varphi}}\right)\left(1-\frac{h \sin \varphi}{2 R_{0}}\right) \\
& I_{2}=\sum_{k=1}^{l} \int_{\zeta_{k}}^{\zeta_{k+1}} \rho^{(k)} \zeta^{2}\left(1+\frac{\zeta}{R_{\varphi}}\right)\left(1+\frac{\zeta \sin \varphi}{R_{0}}\right) \mathrm{d} \zeta+\frac{1}{3} \rho_{F}^{+} h_{F}^{+} \frac{h^{2}}{4}\left(1+\frac{h}{2 R_{\varphi}}\right)\left(1+\frac{h \sin \varphi}{2 R_{0}}\right)+\frac{1}{3} \rho_{F}^{-} h_{F}^{-} \frac{h^{2}}{4}\left(1-\frac{h}{2 R_{\varphi}}\right)\left(1-\frac{h \sin \varphi}{2 R_{0}}\right)
\end{aligned}
$$

are the mass inertias and $\rho^{(k)}$ is the mass density of the material per unit of volume of the $k$-th ply, while $\rho_{F}^{+}, h_{F}^{+}$ and $\rho_{F}^{-}, h_{F}^{-}$are the mass density of the material per unit of volume and the thickness of the elastic foundation at the top and the bottom surface of the shell, respectively. The first three equations (23) represent translational equilibrium along meridian $\varphi$, circumferential $s$ and normal $\zeta$ directions, while the last two are rotational equi- librium equations about the $s$ and $\varphi$ directions, respectively. Furthermore, the generalized external forces $q_{\varphi}, q_{s}, q_{n}, m_{\varphi}, m_{s}$ due to the external forces and the Winkler-Pasternak elastic foundation acting on the top and bottom surfaces of the shell can be evaluated using the static equivalence principle $[12,79,83]$ and can be written on the reference surface of the doubly-curved shell as follows:

$$
\begin{aligned}
q_{\varphi}= & q_{\varphi}^{+}\left(1+\frac{h}{2 R_{\varphi}}\right)^{2}\left(1+\frac{h \sin \varphi}{2 R_{0}}\right)+q_{\varphi}^{-}\left(1-\frac{h}{2 R_{\varphi}}\right)^{2}\left(1-\frac{h \sin \varphi}{2 R_{0}}\right) \\
& -\left(k_{\varphi}^{+}\left(1+\frac{h}{2 R_{\varphi}}\right)^{3}\left(1+\frac{h \sin \varphi}{2 R_{0}}\right)+k_{\varphi}^{-}\left(1-\frac{h}{2 R_{\varphi}}\right)^{3}\left(1-\frac{h \sin \varphi}{2 R_{0}}\right)\right) u_{\varphi} \\
& -\frac{h}{2}\left(k_{\varphi}^{+}\left(1+\frac{h}{2 R_{\varphi}}\right)^{2}\left(1+\frac{h \sin \varphi}{2 R_{0}}\right)-k_{\varphi}^{-}\left(1-\frac{h}{2 R_{\varphi}}\right)^{2}\left(1-\frac{h \sin \varphi}{2 R_{0}}\right)\right) \beta_{\varphi} \\
q_{s}= & q_{s}^{+}\left(1+\frac{h}{2 R_{\varphi}}\right)^{2}\left(1+\frac{h \sin \varphi}{2 R_{0}}\right)+q_{s}^{-}\left(1-\frac{h}{2 R_{\varphi}}\right)^{2}\left(1-\frac{h \sin \varphi}{2 R_{0}}\right) \\
& -\left(k_{s}^{+}\left(1+\frac{h}{2 R_{\varphi}}\right)\left(1+\frac{h \sin \varphi}{2 R_{0}}\right)^{3}+k_{s}^{-}\left(1-\frac{h}{2 R_{\varphi}}\right)\left(1-\frac{h \sin \varphi}{2 R_{0}}\right)^{3}\right) u_{s} \\
& -\frac{h}{2}\left(k_{s}^{+}\left(1+\frac{h}{2 R_{\varphi}}\right)\left(1+\frac{h \sin \varphi}{2 R_{0}}\right)^{2}-k_{s}^{-}\left(1-\frac{h}{2 R_{\varphi}}\right)\left(1-\frac{h \sin \varphi}{2 R_{0}}\right)^{2}\right) \beta_{s}
\end{aligned}
$$




$$
\begin{aligned}
& q_{n}=q_{n}^{+}\left(1+\frac{h}{2 R_{\varphi}}\right)\left(1+\frac{h \sin \varphi}{2 R_{0}}\right)+q_{n}^{-}\left(1-\frac{h}{2 R_{\varphi}}\right)\left(1-\frac{h \sin \varphi}{2 R_{0}}\right)-\left(k_{n}^{+}\left(1+\frac{h}{2 R_{\varphi}}\right)\left(1+\frac{h \sin \varphi}{2 R_{0}}\right)+k_{n}^{-}\left(1-\frac{h}{2 R_{\varphi}}\right)\left(1-\frac{h \sin \varphi}{2 R_{0}}\right)\right) w \\
& +G_{F}^{+}\left(\frac{1}{R_{\varphi}^{2}\left(1+\frac{h}{2 R_{\varphi}}\right)^{2}} \frac{\partial^{2} w}{\partial \varphi^{2}}+\left(\frac{\cos \varphi}{R_{\varphi}\left(1+\frac{h}{2 R_{\varphi}}\right) R_{0}\left(1+\frac{h \sin \varphi}{2 R_{0}}\right)}-\frac{1}{R_{\varphi}^{3}\left(1+\frac{h}{2 R_{\varphi}}\right)^{3}} \frac{\partial R_{\varphi}}{\partial \varphi}\right) \frac{\partial w}{\partial \varphi}\right. \\
& \left.+\frac{1}{\left(1+\frac{h \sin \varphi}{2 R_{0}}\right)^{2}} \frac{\partial^{2} w}{\partial s^{2}}\right)\left(1+\frac{h}{2 R_{\varphi}}\right)\left(1+\frac{h \sin \varphi}{2 R_{0}}\right) \\
& +G_{F}^{-}\left(\frac{1}{R_{\varphi}^{2}\left(1-\frac{h}{2 R_{\varphi}}\right)^{2}} \frac{\partial^{2} w}{\partial \varphi^{2}}+\left(\frac{\cos \varphi}{R_{\varphi}\left(1-\frac{h}{2 R_{\varphi}}\right) R_{0}\left(1-\frac{h \sin \varphi}{2 R_{0}}\right)}-\frac{1}{R_{\varphi}^{3}\left(1-\frac{h}{2 R_{\varphi}}\right)^{3}} \frac{\partial R_{\varphi}}{\partial \varphi}\right) \frac{\partial w}{\partial \varphi}\right. \\
& \left.+\frac{1}{\left(1-\frac{h \sin \varphi}{2 R_{0}}\right)^{2}} \frac{\partial^{2} w}{\partial s^{2}}\right)\left(1-\frac{h}{2 R_{\varphi}}\right)\left(1-\frac{h \sin \varphi}{2 R_{0}}\right) \\
& m_{\varphi}=q_{\varphi}^{+} \frac{h}{2}\left(1+\frac{h}{2 R_{\varphi}}\right)\left(1+\frac{h \sin \varphi}{2 R_{0}}\right)-q_{\varphi}^{-} \frac{h}{2}\left(1-\frac{h}{2 R_{\varphi}}\right)\left(1-\frac{h \sin \varphi}{2 R_{0}}\right) \\
& -\frac{h}{2}\left(k_{\varphi}^{+}\left(1+\frac{h}{2 R_{\varphi}}\right)^{2}\left(1+\frac{h \sin \varphi}{2 R_{0}}\right)-k_{\varphi}^{-}\left(1-\frac{h}{2 R_{\varphi}}\right)^{2}\left(1-\frac{h \sin \varphi}{2 R_{0}}\right)\right) u_{\varphi} \\
& -\frac{h^{2}}{4}\left(k_{\varphi}^{+}\left(1+\frac{h}{2 R_{\varphi}}\right)\left(1+\frac{h \sin \varphi}{2 R_{0}}\right)+k_{\varphi}^{-}\left(1-\frac{h}{2 R_{\varphi}}\right)\left(1-\frac{h \sin \varphi}{2 R_{0}}\right)\right) \beta_{\varphi} \\
& m_{s}=q_{s}^{+} \frac{h}{2}\left(1+\frac{h}{2 R_{\varphi}}\right)\left(1+\frac{h \sin \varphi}{2 R_{0}}\right)-q_{s}^{-} \frac{h}{2}\left(1-\frac{h}{2 R_{\varphi}}\right)\left(1-\frac{h \sin \varphi}{2 R_{0}}\right) \\
& -\frac{h}{2}\left(k_{s}^{+}\left(1+\frac{h}{2 R_{\varphi}}\right)\left(1+\frac{h \sin \varphi}{2 R_{0}}\right)^{2}-k_{s}^{-}\left(1-\frac{h}{2 R_{\varphi}}\right)\left(1-\frac{h \sin \varphi}{2 R_{0}}\right)^{2}\right) u_{s} \\
& -\frac{h^{2}}{4}\left(k_{s}^{+}\left(1+\frac{h}{2 R_{\varphi}}\right)\left(1+\frac{h \sin \varphi}{2 R_{0}}\right)+k_{s}^{-}\left(1-\frac{h}{2 R_{\varphi}}\right)\left(1-\frac{h \sin \varphi}{2 R_{0}}\right)\right) \beta_{s}
\end{aligned}
$$

where $q_{\varphi}^{+}, q_{\varphi}^{-}, q_{s}^{+}, q_{s}^{-}, q_{n}^{+}, q_{n}^{-}$are the external forces and $k_{\varphi}^{+}, k_{\varphi}^{-}, k_{s}^{+}, k_{s}^{-}, k_{n}^{+}, k_{n}^{-}$are the Winkler elastic stiffnesses in the three principal directions $\varphi, s, \zeta$ at the top and the bottom surface of the shell, respectively; $G_{F}^{+}, G_{F}^{-}$are the shear modulus of the Pasternak elastic foundation at the top and the bottom surface of the shell. It is worth noting that the formulation of the Winkler-Pasternak foundation is based on the first-order approximated assumption that the foundation is a homogeneous material of uniform thickness $h_{F}^{+}, h_{F}^{-}[12,79,83]$. Furthermore, as additional hypothesis, the effect of the damping of the foundation is neglected. The three basic set of equations, 
namely the kinematic (18), constitutive (19) and motion (23) equations may be combined to give the fundamental system of equations, also known as the governing system of equations. By replacing the kinematic equations (18) into the constitutive equations (19) and the result of this substitution into the motion equations (23), the complete equations of motion in terms of displacements can be written as:

$$
\begin{aligned}
& {\left[\begin{array}{lllll}
L_{11} & L_{12} & L_{13} & L_{14} & L_{15} \\
L_{21} & L_{22} & L_{23} & L_{24} & L_{25} \\
L_{31} & L_{32} & L_{33} & L_{34} & L_{35} \\
L_{41} & L_{42} & L_{43} & L_{44} & L_{45} \\
L_{51} & L_{52} & L_{53} & L_{54} & L_{55}
\end{array}\right]\left[\begin{array}{c}
u_{\varphi} \\
u_{s} \\
w \\
\beta_{\varphi} \\
\beta_{s}
\end{array}\right]+\left[\begin{array}{c}
q_{\varphi} \\
q_{s} \\
q_{n} \\
m_{\varphi} \\
m_{s}
\end{array}\right]} \\
& =\left[\begin{array}{ccccc}
I_{0 \varphi} & 0 & 0 & I_{1 \varphi} & 0 \\
0 & I_{0 s} & 0 & 0 & I_{1 s} \\
0 & 0 & I_{0} & 0 & 0 \\
I_{1 \varphi} & 0 & 0 & I_{2} & 0 \\
0 & I_{1 s} & 0 & 0 & I_{2}
\end{array}\right]\left[\begin{array}{c}
\ddot{u}_{\varphi} \\
\ddot{u}_{s} \\
\ddot{w} \\
\ddot{\beta}_{\varphi} \\
\ddot{\beta}_{s}
\end{array}\right]
\end{aligned}
$$

where $L_{i j}, i, j=1, \cdots, 5$ are the equilibrium operators and the new mass inertias are defined as follows:

$$
\begin{aligned}
& I_{0 \varphi}=I_{0}+\frac{2 I_{1}}{R_{\varphi}}+\frac{I_{2}}{R_{\varphi}^{2}}, I_{1 \varphi}=I_{1}+\frac{I_{2}}{R_{\varphi}} \\
& I_{0 s}=I_{0}+\frac{2 I_{1} \sin \varphi}{R_{0}}+\frac{I_{2} \sin ^{2} \varphi}{R_{0}^{2}}, I_{1 s}=I_{1}+\frac{I_{2} \sin \varphi}{R_{0}}
\end{aligned}
$$

It is worth noting that, differently from previous works by Tornabene [77,78], the mass matrix and the equilibrium operators $L_{i j}$, introduced in Equation (27), have changed due to the choice of using the new kinematical model (18). Furthermore, the second derivative of the principal radius $R_{\varphi}(\varphi)$ respect to $\varphi$ has to be evaluated, as it can be inferred from the explicit form of the equilibrium operators $L_{i j}$. Three types of boundary conditions are considered, namely the fully clamped edge boundary condition $(C)$, soft simply supported edge boundary conditions $(S)$ and the free edge boundary condition $(F)$. The equations describing the boundary conditions can be written as follows:

Clamped edge boundary conditions (C)

$$
\begin{aligned}
& u_{\varphi}=u_{s}=w=\beta_{\varphi}=\beta_{s}=0 \\
& \text { at } \varphi=\varphi_{0} \text { or } \varphi=\varphi_{1}, 0 \leq s \leq s_{0} \\
& u_{\varphi}=u_{s}=w=\beta_{\varphi}=\beta_{s}=0 \\
& \text { at } s=0 \text { or } s=s_{0}, \varphi_{0} \leq \varphi \leq \varphi_{1}
\end{aligned}
$$

Soft simply supported edge boundary conditions $(S)$

$$
\begin{aligned}
& u_{s}=w=\beta_{s}=0, N_{\varphi}+\frac{M_{\varphi}}{R_{\varphi}}=M_{\varphi}=0 \\
& \text { at } \varphi=\varphi_{0} \text { or } \varphi=\varphi_{1}, 0 \leq s \leq s_{0}
\end{aligned}
$$

$$
\begin{aligned}
& u_{\varphi}=w=\beta_{\varphi}=0, N_{s}+\frac{M_{s}}{R_{s}}=M_{s}=0 \\
& \text { at } s=0 \text { or } s=s_{0}, \varphi_{0} \leq \varphi \leq \varphi_{1}
\end{aligned}
$$

Free edge boundary conditions $(F)$

$$
\begin{aligned}
& N_{\varphi}+\frac{M_{\varphi}}{R_{\varphi}}=N_{\varphi s}+M_{\varphi s} \frac{\sin \varphi}{R_{0}}=T_{\varphi}=M_{\varphi}=M_{\varphi s}=0 \\
& \text { at } \varphi=\varphi_{0} \text { or } \varphi=\varphi_{1}, 0 \leq s \leq s_{0} \\
& N_{s}+M_{s} \frac{\sin \varphi}{R_{0}}=N_{s \varphi}+\frac{M_{s \varphi}}{R_{\varphi}}=T_{s}=M_{s}=M_{s \varphi}=0 \\
& \text { at } s=0 \text { or } s=s_{0}, \varphi_{0} \leq \varphi \leq \varphi_{1}
\end{aligned}
$$

where $\varphi_{0}=\hat{\varphi}_{1}$ and $\varphi_{1}=\hat{\varphi}_{N}$. In addition to the external boundary conditions, the kinematic and physical compatibility conditions should be satisfied at the common closing meridians with $s=0,2 \pi R_{0}$, if a complete shell of revolution is considered. The kinematic compatibility conditions include the continuity of displacements. The physical compatibility conditions can be only represented by the five continuous conditions for the generalized stress resultants. To consider complete revolute shells, it is necessary to implement the kinematic and physical compatibility conditions between the two computational meridians with $s=0$ and with $s_{0}=2 \pi R_{0}$ :

Kinematic compatibility conditions along the closing meridian $\left(s=0,2 \pi R_{0}\right)$

$$
\begin{aligned}
& u_{\varphi}(\varphi, 0, t)=u_{\varphi}\left(\varphi, s_{0}, t\right), u_{s}(\varphi, 0, t)=u_{s}\left(\varphi, s_{0}, t\right), \\
& w(\varphi, 0, t)=w\left(\varphi, s_{0}, t\right), \\
& \beta_{\varphi}(\varphi, 0, t)=\beta_{\varphi}\left(\varphi, s_{0}, t\right), \beta_{s}(\varphi, 0, t) \\
& =\beta_{s}\left(\varphi, s_{0}, t\right) \text { for } \varphi_{0} \leq \varphi \leq \varphi_{1}
\end{aligned}
$$

Physical compatibility conditions along the closing meridian $\left(s=0,2 \pi R_{0}\right)$

$$
\begin{aligned}
& N_{s}(\varphi, 0, t)+M_{s}(\varphi, 0, t) \frac{\sin \varphi}{R_{0}} \\
& =N_{s}\left(\varphi, s_{0}, t\right)+M_{s}\left(\varphi, s_{0}, t\right) \frac{\sin \varphi}{R_{0}}, \\
& N_{s \varphi}(\varphi, 0, t)+\frac{M_{s \varphi}(\varphi, 0, t)}{R_{\varphi}} \\
& =N_{s \varphi}\left(\varphi, s_{0}, t\right)+\frac{M_{s \varphi}\left(\varphi, s_{0}, t\right)}{R_{\varphi}}, \\
& T_{s}(\varphi, 0, t)=T_{s}\left(\varphi, s_{0}, t\right), M_{s}(\varphi, 0, t)=M_{s}\left(\varphi, s_{0}, t\right), \\
& M_{s \varphi}(\varphi, 0, t)=M_{s \varphi}\left(\varphi, s_{0}, t\right) \text { for } \varphi_{0} \leq \varphi \leq \varphi_{1}
\end{aligned}
$$

where $\varphi_{0}=\hat{\varphi}_{1}$ and $\varphi_{1}=\hat{\varphi}_{N}$. In an analogous way, in order to consider a toroidal shell of revolution, it is necessary to implement the kinematic and physical compatibility conditions between the two computational parallels 
with $\varphi_{0}=0$ and with $\varphi_{1}=2 \pi$ :

Kinematic compatibility conditions along the closing parallel $(\varphi=0,2 \pi)$

$$
\begin{aligned}
& u_{\varphi}(0, s, t)=u_{\varphi}(2 \pi, s, t), u_{s}(0, s, t)=u_{s}(2 \pi, s, t), \\
& w(0, s, t)=w(2 \pi, s, t), \beta_{\varphi}(0, s, t)=\beta_{\varphi}(2 \pi, s, t), \\
& \beta_{s}(0, s, t)=\beta_{s}(2 \pi, s, t) \text { for } 0 \leq s \leq s_{0}
\end{aligned}
$$

Physical compatibility conditions along the closing $\operatorname{parallel}(\varphi=0,2 \pi)$

$$
\begin{aligned}
& N_{\varphi}(0, s, t)+\frac{M_{\varphi}(0, s, t)}{R_{\varphi}} \\
& =N_{\varphi}(2 \pi, s, t)+\frac{M_{\varphi}(2 \pi, s, t)}{R_{\varphi}}, \\
& N_{\varphi s}(0, s, t)+M_{\varphi s}(0, s, t) \frac{\sin \varphi}{R_{0}} \\
& =N_{\varphi s}(2 \pi, s, t)+M_{\varphi s}(2 \pi, s, t) \frac{\sin \varphi}{R_{0}}, \\
& T_{\varphi}(0, s, t)=T_{\varphi}(2 \pi, s, t), M_{\varphi}(0, s, t)=M_{\varphi}(2 \pi, s, t), \\
& M_{\varphi s}(0, s, t)=M_{\varphi s}(2 \pi, s, t) \text { for } 0 \leq s \leq s_{0}
\end{aligned}
$$

\section{Numerical Implementation}

The Generalized Differential Quadrature method will be used to discretize the derivatives in the governing equations in terms of displacements as well as boundary and compatibility conditions. Since a review of the GDQ Method is presented in Tornabene $[65,83]$, the same approach is used in the present work about the GDQ technique. The Chebyshev-Gauss-Lobatto (C-G-L) grid distribution is assumed. Since the co-ordinates of the grid points of the reference surface in the $\varphi$ direction are introduced in Equation (9), then the co-ordinates of the grid points in the $s$ direction are the following:

$$
\begin{aligned}
& s_{j}=\left(1-\cos \left(\frac{j-1}{M-1} \pi\right)\right) \frac{s_{0}}{2} \\
& \text { for } j=1,2, \cdots, M, \text { for } s \in\left[0, s_{0}\right]\left(\text { with } s \leq \vartheta R_{0}\right)
\end{aligned}
$$

where $M$ is the total number of sampling points used to discretize the domain in $s$ direction of the doublycurved shell. It has been proven that for the Lagrange interpolating polynomials, the Chebyshev-Gauss-Lobatto sampling points rule guarantees convergence and efficiency to the GDQ technique $[57-59,63,83]$. For the static analysis, when the inertias are set to zero, the GDQ procedure enables to write the equations of equilibrium (26) in discrete form, transforming each space derivative into a weighted sum of node values of independent variables. Each approximated equation is valid in a single sampling point. Thus, the whole system of differential equations has been discretized and the global assembling leads to the following set of linear algebraic equations:

$$
\left[\begin{array}{l|l}
\boldsymbol{K}_{b b} & \boldsymbol{K}_{b d} \\
\hline \boldsymbol{K}_{d b} & \boldsymbol{K}_{d d}
\end{array}\right]\left[\begin{array}{l}
\boldsymbol{\delta}_{b} \\
\boldsymbol{\delta}_{d}
\end{array}\right]=\left[\begin{array}{l}
\boldsymbol{f}_{b} \\
\boldsymbol{f}_{d}
\end{array}\right]
$$

In the above mentioned matrices and vectors, the partitioning is set forth by subscripts $b$ and $d$, referring to the system degrees of freedom and standing for boundary and domain, respectively. In this sense, $b$-equations represent the discrete boundary conditions, which are valid only for the points lying on constrained edges of the shell; while $d$-equations are the equilibrium equations, assigned to the interior nodes. In order to make the computation more efficient, static condensation of non-domain degrees of freedom is performed:

$$
\left(\boldsymbol{K}_{d d}-\boldsymbol{K}_{d b} \boldsymbol{K}_{b b}^{-1} \boldsymbol{K}_{b d}\right) \boldsymbol{\delta}_{d}=\boldsymbol{f}_{d}-\boldsymbol{K}_{d b} \boldsymbol{K}_{b b}^{-1} \boldsymbol{f}_{b}
$$

The deflection of the structures considered can be determined by solving the linear algebraic problem (40). In particular, the solution procedure by means of the GDQ technique has been implemented in a MATLAB code. Otherwise, when the external forces $q_{\varphi}^{+}, q_{\varphi}^{-}, q_{s}^{+}, q_{s}^{-}, q_{n}^{+}, q_{n}^{-}$ are set to zero, the free vibration of laminated composite doubly-curved shells and panels of revolution can be studied. Using the method of variable separation, it is possible to seek solutions that are harmonic in time and whose frequency is $f=\omega / 2 \pi$. The displacement field can be written as follows:

$$
\begin{aligned}
& u_{\varphi}(\varphi, s, t)=U^{\varphi}(\varphi, s) \mathrm{e}^{i \omega t} \\
& u_{s}(\varphi, s, t)=U^{s}(\varphi, s) \mathrm{e}^{i \omega t} \\
& w(\varphi, s, t)=W(\varphi, s) \mathrm{e}^{i \omega t} \\
& \beta_{\varphi}(\varphi, s, t)=B^{\varphi}(\varphi, s) \mathrm{e}^{i \omega t} \\
& \beta_{s}(\varphi, s, t)=B^{s}(\varphi, s) \mathrm{e}^{i \omega t}
\end{aligned}
$$

where the vibration spatial amplitude values $U^{\varphi}, U^{s}, W$, $B^{\varphi}, B^{s}$ fulfill the fundamental differential system. Thus, the whole system of differential equations has been discretized and the global assembling leads to the following linear eigenvalue problem:

$$
\left[\begin{array}{c|c}
\boldsymbol{K}_{b b} & \boldsymbol{K}_{b d} \\
\hline \boldsymbol{K}_{d b} & \boldsymbol{K}_{d d}
\end{array}\right]\left[\begin{array}{l}
\boldsymbol{\delta}_{b} \\
\hline \boldsymbol{\delta}_{d}
\end{array}\right]=\omega^{2}\left[\begin{array}{c|c}
\boldsymbol{0} & \boldsymbol{0} \\
\hline \boldsymbol{0} & \boldsymbol{M}_{d d}
\end{array}\right]\left[\begin{array}{l}
\boldsymbol{\delta}_{b} \\
\hline \boldsymbol{\delta}_{d}
\end{array}\right]
$$

In order to make the computation more efficient, kinematic condensation of non-domain degrees of freedom is performed:

$$
\left(\boldsymbol{K}_{d d}-\boldsymbol{K}_{d b}\left(\boldsymbol{K}_{b b}\right)^{-1} \boldsymbol{K}_{b d}\right) \boldsymbol{\delta}_{d}=\omega^{2} \boldsymbol{M}_{d d} \boldsymbol{\delta}
$$

The natural frequencies of the structure considered can be determined by solving the standard eigenvalue problem (43). In particular, the solution procedure by means 
of the GDQ technique has been implemented in a MATLAB code. Finally, the results in terms of frequencies are obtained using the eigs function of MATLAB software. More details regarding the way to obtained the Equations (40) and (43) can be found in the previous works [57-59,63,65-68,83]. It is worth noting that, with the present approach, differing from the finite element method, no integration occurs prior to the global assembly of the linear system, and this approach leads to a further computational cost saving in favor of the Differential Quadrature technique.

\section{Numerical Results}

In the present paragraph, some results and considerations about the static analysis and the free vibration problem of laminated composite doubly-curved shells and panels of revolution are presented. The analysis has been carried out by means of numerical procedures illustrated above. One of the aims of this paper is to compare results obtained through the GDQ method with the ones obtained through finite element techniques. In order to verify the accuracy of the present method, some comparisons and tests have been performed. Extensive attempts to validate the numerical procedure have been made for the isotropic and anisotropic cases and can be found in the $\mathrm{Ph} . \mathrm{D}$. Thesis by Tornabene [57] and in the book by Tornabene [83]. In this work, the static deflection and the frequency parameters evaluated by the present formulation are in good agreement with the results obtained with the finite ele- ment method. The geometrical boundary conditions for a panel are identified by the following convention. For example, symbolism CSCF shows that the edges $\varphi=\varphi_{0 d}, s=0, \varphi=\varphi_{1}, s=s_{0}$ are clamped, simply supported, clamped and free, respectively. On the contrary, for a complete shell of revolution or for a toroidal shell, symbolism CF shows that the edges $\varphi=\varphi_{0}$ and $\varphi=\varphi_{1}$ or $s=0$ and $s=s_{0}$ are clamped and free, respectively. The missing boundary conditions are the kinematic and physical compatibility conditions that are applied at the same closing meridians for $s=0$ and $s_{0}=2 \pi R_{0}$ or at the same closing parallels for $\varphi=0$ and $\varphi=2 \pi$, respectively. Table 1 presents the static deflection at the point $A=\left(90^{\circ}, 0^{\circ}\right)$ for a SSSS spherical panel resting on elastic foundation and subjected to a uniformly distributed load $q_{n}^{+}=-10000 \mathrm{~Pa}$ at the top surface by considering different lamination schemes. As can be seen, the numerical results show an excellent agreement for all the cases considered. GDQ results are compared with the FEM results obtained with Straus 7 commercial software using 8 node parabolic shell elements. In order to illustrate the effect of the foundation Figures $\mathbf{4}$ and $\mathbf{5}$ show the stress resultants for a $(30 / 45 / 70)$ CCCC spherical panel subjected to a uniformly distributed load $q_{n}^{+}=-10000 \mathrm{~Pa}$ at the top surface. Figure 4 illustrates the stress resultants obtained without considering the foundation, while Figure 5 presents the same quantities obtained considering the elastic foundation. The geometrical and material properties are the same reported in

Table 1. Static deflection for a SSSS spherical panel at the point $A=\left(90^{\circ}, 0^{\circ}\right)$ resting on elastic foundation and subjected to a uniformly distributed load $q_{n}^{+}=-10000 \mathrm{~Pa}$ at the top surface.

\begin{tabular}{|c|c|c|c|c|c|c|}
\hline \multirow[b]{3}{*}{ Lamination scheme } & \multicolumn{6}{|c|}{ Foundation properties: $\rho_{F}^{-}=0 \mathrm{~kg} / \mathrm{m}^{3}, h_{F}^{-}=0 \mathrm{~m}, G_{F}^{-}=0 \mathrm{~N} / \mathrm{m}, k_{\varphi}^{-}=k_{s}^{-}=0 \mathrm{~N} / \mathrm{m}^{3}$} \\
\hline & \multicolumn{2}{|c|}{$k_{n}^{-}=0 \mathrm{~N} / \mathrm{m}^{3}$} & \multicolumn{2}{|c|}{$k_{n}^{-}=1.5 \times 10^{7} \mathrm{~N} / \mathrm{m}^{3}$} & \multicolumn{2}{|c|}{$k_{n}^{-}=7.5 \times 10^{7} \mathrm{~N} / \mathrm{m}^{3}$} \\
\hline & $\begin{array}{c}\text { GDQ-GST } \\
31 \times 31\end{array}$ & $\begin{array}{c}\text { Straus } \\
100 \times 100(8 \text { nodes })\end{array}$ & $\begin{array}{c}\text { GDQ-GST } \\
31 \times 31\end{array}$ & $\begin{array}{c}\text { Straus } \\
100 \times 100(8 \text { nodes })\end{array}$ & $\begin{array}{c}\text { GDQ-GST } \\
31 \times 31\end{array}$ & $\begin{array}{c}\text { Straus } \\
100 \times 100(8 \text { nodes })\end{array}$ \\
\hline Isotropic & $-1.570 \mathrm{E}-04$ & $-1.571 \mathrm{E}-04$ & $-1.275 \mathrm{E}-04$ & $-1.274 \mathrm{E}-04$ & $-7.281 \mathrm{E}-05$ & $-7.246 \mathrm{E}-05$ \\
\hline$(0 / 90)$ & $-5.911 \mathrm{E}-04$ & $-5.862 \mathrm{E}-04$ & $-3.257 \mathrm{E}-04$ & $-3.229 \mathrm{E}-04$ & $-1.129 \mathrm{E}-04$ & $-1.119 \mathrm{E}-04$ \\
\hline$(0 / 90 / 0)$ & $-5.922 \mathrm{E}-04$ & $-5.875 \mathrm{E}-04$ & $-3.263 \mathrm{E}-04$ & $-3.235 \mathrm{E}-04$ & $-1.131 \mathrm{E}-04$ & $-1.121 \mathrm{E}-04$ \\
\hline$(0 / 90)_{a s}$ & $-5.911 \mathrm{E}-04$ & $-5.863 \mathrm{E}-04$ & $-3.263 \mathrm{E}-04$ & $-3.235 \mathrm{E}-04$ & $-1.130 \mathrm{E}-04$ & $-1.120 \mathrm{E}-04$ \\
\hline$(0 / 90)_{s}$ & $-5.909 \mathrm{E}-04$ & $-5.862 \mathrm{E}-04$ & $-3.264 \mathrm{E}-04$ & $-3.236 \mathrm{E}-04$ & $-1.130 \mathrm{E}-04$ & $-1.120 \mathrm{E}-04$ \\
\hline$(30)$ & $-4.589 \mathrm{E}-04$ & $-4.418 \mathrm{E}-04$ & $-2.503 \mathrm{E}-04$ & $-2.444 \mathrm{E}-04$ & $-9.899 \mathrm{E}-05$ & $-9.758 \mathrm{E}-05$ \\
\hline$(30 / 45)$ & $-4.476 \mathrm{E}-04$ & $-4.276 \mathrm{E}-04$ & $-2.303 \mathrm{E}-04$ & $-2.237 \mathrm{E}-04$ & $-9.102 \mathrm{E}-05$ & $-8.940 \mathrm{E}-05$ \\
\hline$(30 / 45 / 70)$ & $-4.413 \mathrm{E}-04$ & $-4.253 \mathrm{E}-04$ & $-2.420 \mathrm{E}-04$ & $-2.361 \mathrm{E}-04$ & $-9.507 \mathrm{E}-05$ & $-9.351 \mathrm{E}-05$ \\
\hline$(30 / 45 / 70 / 90)$ & $-3.859 \mathrm{E}-04$ & $-3.764 \mathrm{E}-04$ & $-2.364 \mathrm{E}-04$ & $-2.319 \mathrm{E}-04$ & $-9.686 \mathrm{E}-05$ & $-9.553 \mathrm{E}-05$ \\
\hline
\end{tabular}

${ }^{*}$ Geometric characteristics: $R_{\varphi}=R_{s}=10 \mathrm{~m}, \quad R_{b}=0 \mathrm{~m}, \varphi \in\left[60^{\circ}, 120^{\circ}\right], \vartheta \in\left[-30^{\circ}, 30^{\circ}\right], \quad h=0.09 \mathrm{~m}$, Isotropic material: $E=73 \mathrm{GPa}, \quad v=0.3$, $\rho=2700 \mathrm{~kg} / \mathrm{m}^{3}$, Orthotropic material: $E_{1}=137.9 \mathrm{GPa}, E_{2}=8.96 \mathrm{GPa}, G_{12}=G_{13}=7.1 \mathrm{GPa}, G_{23}=6.21 \mathrm{GPa}, v_{12}=0.3, \rho=1450 \mathrm{~kg} / \mathrm{m}^{3}$. 

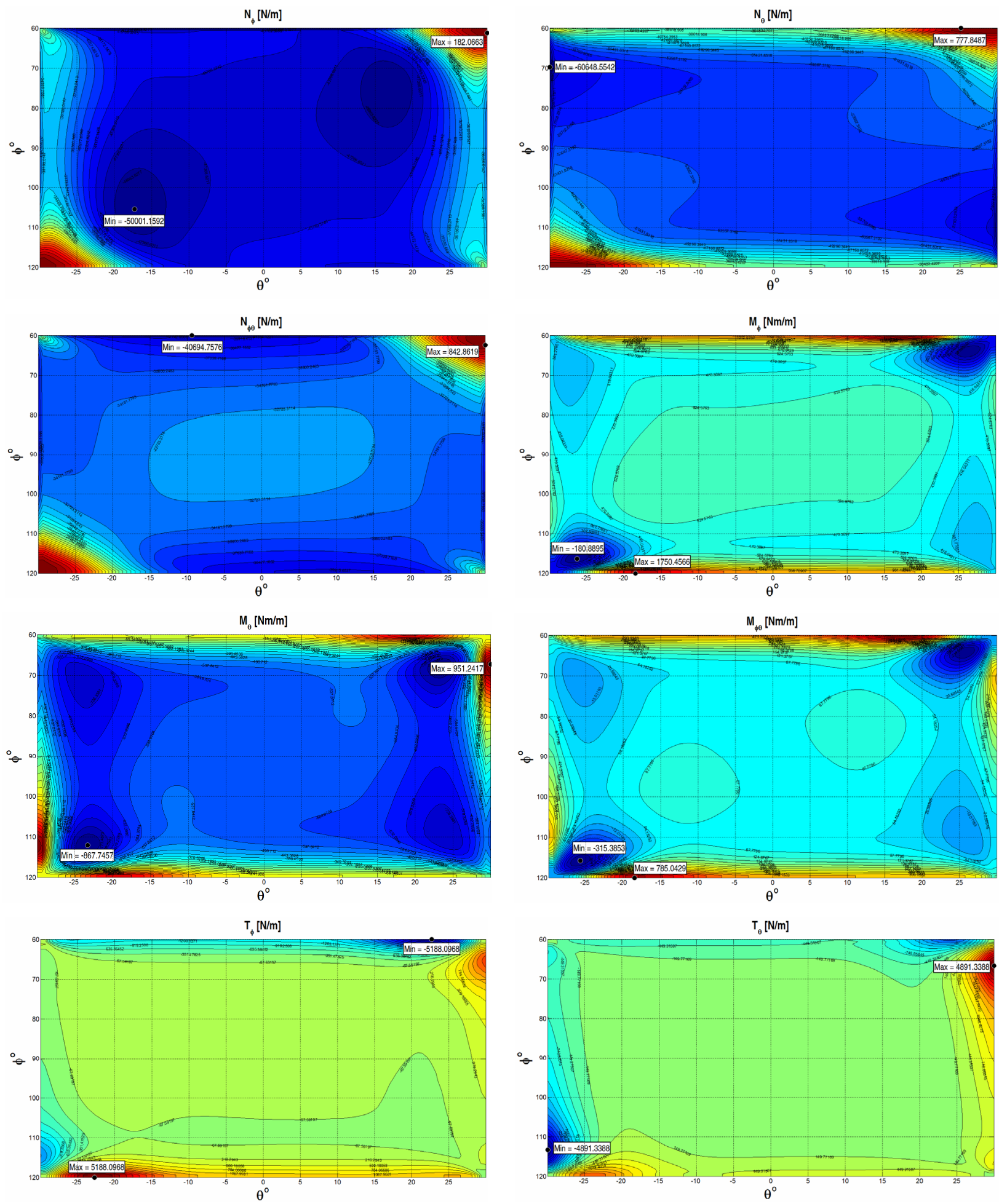

Figure 4. Stress resultants for a $(30 / 45 / 70)$ CCCC spherical panel with a uniformly distributed load $q_{n}^{+}=-10000 \mathrm{~Pa}$ at the top surface and without elastic foundation.

Table 1. As can be seen from the figures, the effect of the foundation reduces the stress resultants as expected. In Tables 2-4, the results, in terms of first ten frequencies obtained by the GDQ Method for the General Shell
Theory (GST) presented above with and without elastic foundations, are compared with the FEM results obtained with Straus 7 commercial software. The details regarding the geometry and the material properties are reported in 

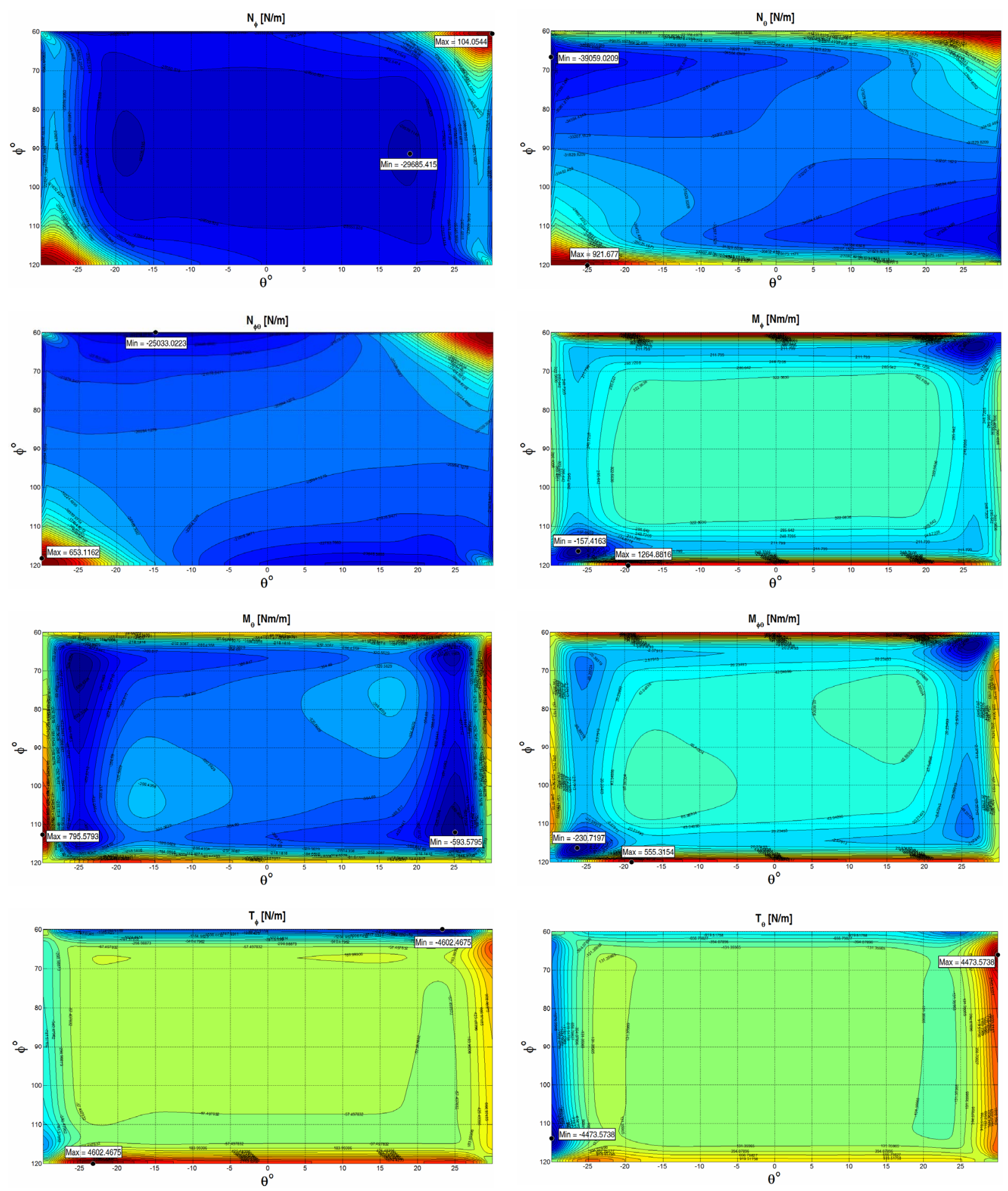

Figure 5. Stress resultants for a $(30 / 45 / 70)$ CCCC spherical panel with a uniformly distributed load $q_{n}^{+}=-10000 \mathrm{~Pa}$ at the top surface resting on Winkler-Pasternak elastic foundation: $\boldsymbol{k}_{n}^{-}=7.5 \times 10^{7} \mathrm{~N} / \mathrm{m}^{3}$ and $\rho_{F}^{-}=h_{F}^{-}=G_{F}^{-}=k_{\varphi}^{-}=k_{s}^{-}=0$.

tables. For the present GDQ results, the grid distributions (9) and (38) with $N=M=31$ have been considered. Tables 2-4 present the first ten frequencies for a SSSS spherical panel characterized by $(0 / 90),(0 / 90 / 0)$ and
(30/45/70) lamination scheme, respectively. As can be seen, the numerical results from the GDQ methodology are very close to those obtained by the commercial program and show an excellent agreement. Tables 5-7 pre- 
Table 2. First ten frequencies for a $(0 / 90)$ SSSS spherical panel resting on elastic foundation.

\begin{tabular}{|c|c|c|c|c|c|c|}
\hline \multirow{3}{*}{$\begin{array}{l}\text { Mode } \\
{[\mathrm{Hz}]}\end{array}$} & \multicolumn{6}{|c|}{ Foundation properties: $\rho_{F}^{-}=0 \mathrm{~kg} / \mathrm{m}^{3}, h_{F}^{-}=0 \mathrm{~m}, G_{F}^{-}=0 \mathrm{~N} / \mathrm{m}, k_{\varphi}^{-}=k_{s}^{-}=0 \mathrm{~N} / \mathrm{m}^{3}$} \\
\hline & \multicolumn{2}{|c|}{$k_{n}^{-}=0 \mathrm{~N} / \mathrm{m}^{3}$} & \multicolumn{2}{|c|}{$k_{n}^{-}=1.5 \times 10^{7} \mathrm{~N} / \mathrm{m}^{3}$} & \multicolumn{2}{|c|}{$k_{n}^{-}=7.5 \times 10^{7} \mathrm{~N} / \mathrm{m}^{3}$} \\
\hline & $\begin{array}{c}\text { GDQ-GST } \\
31 \times 31\end{array}$ & $\begin{array}{c}\text { Straus } \\
100 \times 100(8 \text { nodes })\end{array}$ & $\begin{array}{c}\text { GDQ-GST } \\
31 \times 31\end{array}$ & $\begin{array}{c}\text { Straus } \\
100 \times 100(8 \text { nodes })\end{array}$ & $\begin{array}{c}\text { GDQ-GST } \\
31 \times 31\end{array}$ & $\begin{array}{c}\text { Straus } \\
100 \times 100(8 \text { nodes })\end{array}$ \\
\hline$f_{1}$ & 59.775 & 60.016 & 77.703 & 78.035 & 96.630 & 97.056 \\
\hline$f_{2}$ & 64.413 & 64.661 & 83.209 & 83.552 & 111.257 & 111.754 \\
\hline$f_{3}$ & 69.263 & 69.518 & 87.167 & 87.516 & 125.527 & 126.086 \\
\hline$f_{4}$ & 69.527 & 69.790 & 87.285 & 87.604 & 134.209 & 134.795 \\
\hline$f_{5}$ & 70.561 & 70.777 & 87.554 & 87.908 & 135.981 & 136.554 \\
\hline$f_{6}$ & 70.612 & 70.866 & 88.298 & 88.644 & 136.490 & 137.085 \\
\hline$f_{7}$ & 73.148 & 73.425 & 89.717 & 90.078 & 137.712 & 138.301 \\
\hline$f_{8}$ & 75.950 & 76.275 & 92.796 & 93.200 & 137.805 & 138.399 \\
\hline$f_{9}$ & 76.739 & 76.996 & 93.436 & 93.784 & 137.961 & 138.549 \\
\hline$f_{10}$ & 79.349 & 79.641 & 95.537 & 95.914 & 141.355 & 141.981 \\
\hline
\end{tabular}

*Geometric characteristics: $R_{\varphi}=R_{s}=10 \mathrm{~m}, \quad R_{b}=0 \mathrm{~m}, \varphi \in\left[60^{\circ}, 120^{\circ}\right], \vartheta \in\left[-30^{\circ}, 30^{\circ}\right], \quad h=0.09 \mathrm{~m}$, Orthotropic material: $E_{1}=137.9 \mathrm{GPa}$, $E_{2}=8.96 \mathrm{GPa}, G_{12}=G_{13}=7.1 \mathrm{GPa}, G_{23}=6.21 \mathrm{GPa}, v_{12}=0.3, \rho=1450 \mathrm{~kg} / \mathrm{m}^{3}$.

Table 3. First ten frequencies for a $(\mathbf{0} / 90 / 0)$ SSSS spherical panel resting on elastic foundation.

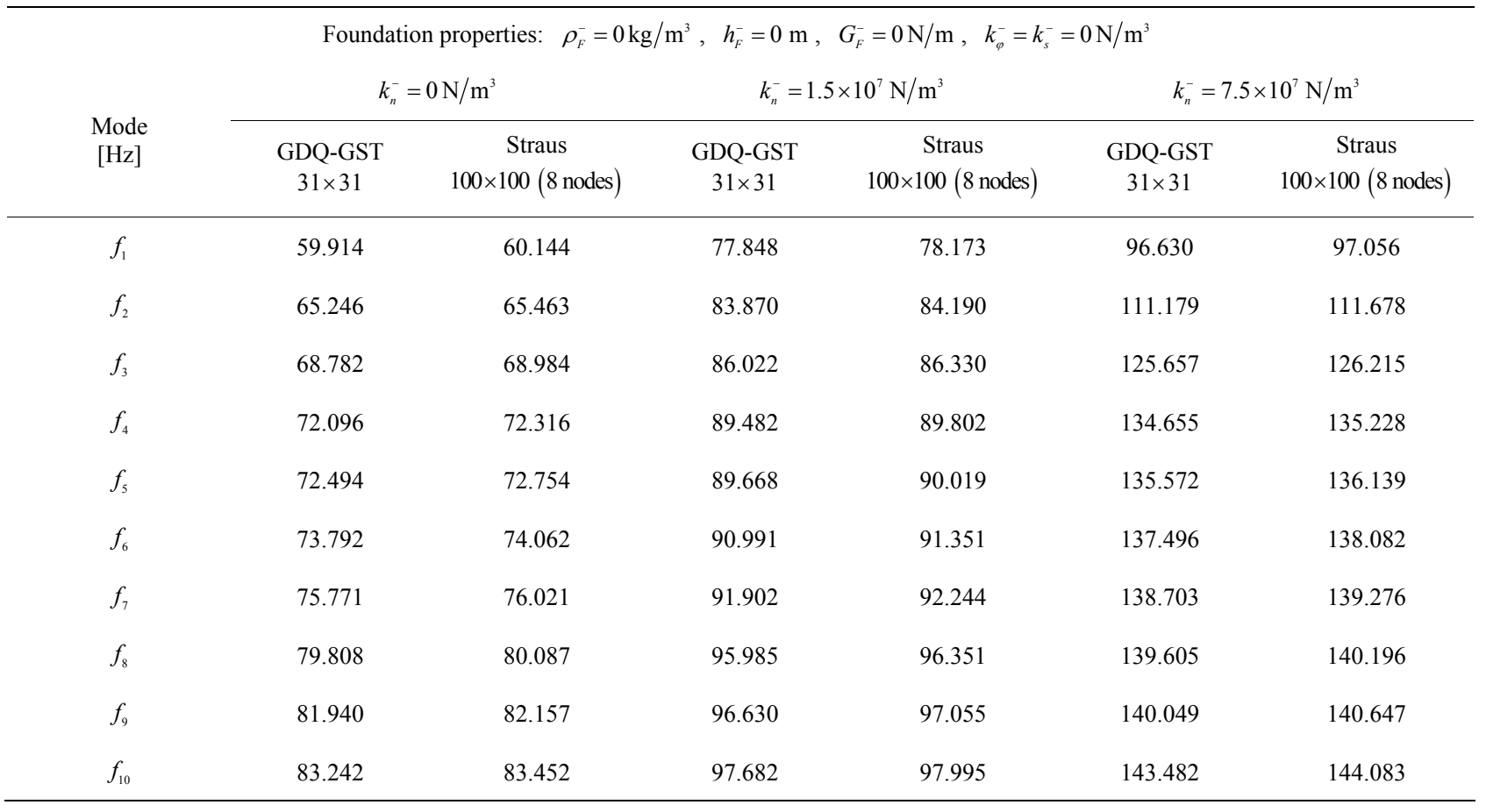

*Geometric characteristics: $R_{\varphi}=R_{s}=10 \mathrm{~m}, \quad R_{b}=0 \mathrm{~m}, \varphi \in\left[60^{\circ}, 120^{\circ}\right], \quad \vartheta \in\left[-30^{\circ}, 30^{\circ}\right], \quad h=0.09 \mathrm{~m}$, Orthotropic material: $E_{1}=137.9 \mathrm{GPa}$, $E_{2}=8.96 \mathrm{GPa}, G_{12}=G_{13}=7.1 \mathrm{GPa}, G_{23}=6.21 \mathrm{GPa}, v_{12}=0.3, \rho=1450 \mathrm{~kg} / \mathrm{m}^{3}$. 
Table 4. First ten frequencies for a $(30 / 45 / 70)$ SSSS spherical panel resting on elastic foundation.

\begin{tabular}{|c|c|c|c|c|c|c|}
\hline \multirow{3}{*}{$\begin{array}{c}\text { Mode } \\
{[\mathrm{Hz}]}\end{array}$} & \multicolumn{6}{|c|}{ Foundation properties: $\rho_{F}^{-}=0 \mathrm{~kg} / \mathrm{m}^{3}, h_{F}^{-}=0 \mathrm{~m}, G_{F}^{-}=0 \mathrm{~N} / \mathrm{m}, k_{\varphi}^{-}=k_{s}^{-}=0 \mathrm{~N} / \mathrm{m}^{3}$} \\
\hline & \multicolumn{2}{|c|}{$k_{n}^{-}=0 \mathrm{~N} / \mathrm{m}^{3}$} & \multicolumn{2}{|c|}{$k_{n}^{-}=1.5 \times 10^{7} \mathrm{~N} / \mathrm{m}^{3}$} & \multicolumn{2}{|c|}{$k_{n}^{-}=7.5 \times 10^{7} \mathrm{~N} / \mathrm{m}^{3}$} \\
\hline & $\begin{array}{c}\text { GDQ-GST } \\
31 \times 31\end{array}$ & $\begin{array}{c}\text { Straus } \\
100 \times 100(8 \text { nodes })\end{array}$ & $\begin{array}{c}\text { GDQ-GST } \\
31 \times 31\end{array}$ & $\begin{array}{c}\text { Straus } \\
100 \times 100(8 \text { nodes })\end{array}$ & $\begin{array}{c}\text { GDQ-GST } \\
31 \times 31\end{array}$ & $\begin{array}{c}\text { Straus } \\
100 \times 100(8 \text { nodes })\end{array}$ \\
\hline$f_{1}$ & 49.808 & 50.761 & 72.378 & 73.212 & 113.366 & 114.996 \\
\hline$f_{2}$ & 50.298 & 51.252 & 72.504 & 73.353 & 122.924 & 124.126 \\
\hline$f_{3}$ & 58.625 & 59.540 & 78.395 & 79.266 & 126.787 & 127.684 \\
\hline$f_{4}$ & 59.718 & 60.646 & 79.653 & 80.515 & 130.940 & 131.884 \\
\hline$f_{5}$ & 63.879 & 64.983 & 82.107 & 83.137 & 132.074 & 132.994 \\
\hline$f_{6}$ & 65.655 & 66.760 & 84.378 & 85.393 & 134.626 & 135.592 \\
\hline$f_{7}$ & 68.939 & 69.923 & 85.701 & 86.673 & 135.405 & 136.422 \\
\hline$f_{8}$ & 70.901 & 71.688 & 88.598 & 89.372 & 138.339 & 139.210 \\
\hline$f_{9}$ & 75.107 & 76.139 & 91.808 & 92.794 & 139.764 & 140.811 \\
\hline$f_{10}$ & 77.134 & 78.199 & 92.876 & 93.925 & 139.863 & 140.883 \\
\hline
\end{tabular}

${ }^{*}$ Geometric characteristics: $R_{\varphi}=R_{s}=10 \mathrm{~m}, \quad R_{b}=0 \mathrm{~m}, \varphi \in\left[60^{\circ}, 120^{\circ}\right], \quad \vartheta \in\left[-30^{\circ}, 30^{\circ}\right], \quad h=0.09 \mathrm{~m}$, Orthotropic material: $E_{1}=137.9 \mathrm{GPa}$, $E_{2}=8.96 \mathrm{GPa}, G_{12}=G_{13}=7.1 \mathrm{GPa}, G_{23}=6.21 \mathrm{GPa}, v_{12}=0.3, \rho=1450 \mathrm{~kg} / \mathrm{m}^{3}$.

sent new results regarding different anisotropic doublycurved shells and panels of revolution resting on Winkler-Pasternak elastic foundations. Tables 5-7 are divided in different columns where each effect of the elastic foundation is considered. At first, the effect of the Winkler modulus on free vibrations has been investigated and compared to the case without the elastic foundation. As can be seen from results, the Winkler foundation increases the stiffness of the structure. The same effect is shown by introducing the Pasternak shear modulus. In addition to these two effects the inertia of the foundation is considered. As expected, the inertia effect increases the mass of the structure and reduces the frequencies. Finally, the Winkler foundation in meridian and circumferential directions is introduced. As can be inferred, also in this case the stiffness of the structure is increased. Table 5 presents the effects under consideration for the $\mathrm{CC}$ complete shell characterized by the $(30 / 45)$ lamination scheme. In this case, the foundation acts on the top surface of the shell. Differently from previous case, Tables 6 and 7 show the first ten frequencies for the $(0 / 30)$ CCCC laminated composite panel and for the $(30 / 60)$ FC laminated composite shell resting on an elastic foundation acting on the bottom surface. Also for these cases, the effect of the initial curvature is considered by comparing the present General Shell Theory (GST) and the Reissner-Mindlin (RM) theory. Thus, as regarding the influence of the initial curvature, it is worth noting that the difference between the two theories considered is low for all the laminated composite doubly-curved structures analyzed. Finally, in Figures 6 and 7, there are reported the first six mode shapes for some of the structures considered above. In particular, for the complete shells of revolution there are some symmetrical mode shapes due to the symmetry of the problem considered in 3D space. In these cases, the symmetrical mode shapes are summarized in one figure. The mode shapes of all the structures have been evaluated by the authors. By using the authors' MATLAB code, these mode shapes have been reconstructed in three-dimensional view by means of considering the displacement field (17) after solving the eigenvalue problem (43). In addition, Hosseini-Hashemi et al. [86] (see table 3) and Zhao and Liew [87] (see Table 6) have compared their results, obtained with a semi analytical method and produced by a meshless method, with those results presented in the articles by Tornabene [65] and by Tornabene et al. [69], respectively. Since the code used to obtain the previous results by Tornabene [65] and Tornabene et al. [69] is similar to the code used to obtain all the results presented in this paper, the results of the work by Hosseini-Hashemi et al. [86] and by Zhao and Liew [87] represent other proofs of the validity and the accuracy of the present procedure. As shown, the exact results by Hosseini-Hashemi et al. [86] and the numerical results by Zhao and Liew [84] are in good agreement with those reported by Tornabene [65] and by 
Table 5. First ten frequencies for a (30/45) CC shell resting on Winkler-Pasternak elastic foundation.

\begin{tabular}{|c|c|c|c|c|c|c|c|c|c|c|}
\hline \multirow{3}{*}{$\begin{array}{l}\text { Mode } \\
{[\mathrm{Hz}]}\end{array}$} & \multicolumn{2}{|c|}{ Foundation properties: } & \multicolumn{4}{|c|}{$\rho_{F}^{+}=1800 \mathrm{~kg} / \mathrm{m}^{3}, \quad h_{F}^{+}=0.1 \mathrm{~m}, \quad k_{n}^{+}=1.5 \times 10^{7} \mathrm{~N} / \mathrm{m}^{3}$} & \multicolumn{4}{|c|}{$G_{F}^{+}=5 \times 10^{5} \mathrm{~N} / \mathrm{m}, \quad k_{\varphi}^{+}=k_{s}^{+}=0.75 \times 10^{7} \mathrm{~N} / \mathrm{m}^{3}$} \\
\hline & \multicolumn{2}{|c|}{$\begin{array}{l}k_{n}^{+}=0, k_{\varphi}^{+}=0 \\
k_{s}^{+}=0, G_{F}^{+}=0 \\
\rho_{F}^{+}=0, h_{F}^{+}=0\end{array}$} & \multicolumn{2}{|c|}{$\begin{array}{l}k_{n}^{+} \neq 0, k_{\varphi}^{+}=0, \\
k_{s}^{+}=0, G_{F}^{+}=0 \\
\rho_{F}^{+}=0, h_{F}^{+}=0\end{array}$} & \multicolumn{2}{|c|}{$\begin{array}{l}k_{n}^{+} \neq 0, k_{\varphi}^{+}=0, \\
k_{s}^{+}=0, G_{F}^{+} \neq 0 \\
\rho_{F}^{+}=0, h_{F}^{+}=0\end{array}$} & \multicolumn{2}{|c|}{$\begin{array}{l}k_{n}^{+} \neq 0, k_{\varphi}^{+}=0, \\
k_{s}^{+}=0, G_{F}^{+} \neq 0 \\
\rho_{F}^{+} \neq 0, h_{F}^{+} \neq 0\end{array}$} & \multicolumn{2}{|c|}{$\begin{array}{l}k_{n}^{+} \neq 0, k_{\varphi}^{+} \neq 0, \\
k_{s}^{+} \neq 0, G_{F}^{+} \neq 0, \\
\rho_{F}^{+} \neq 0, h_{F}^{+} \neq 0\end{array}$} \\
\hline & GDQ-GST & GDQ-RM & GDQ-GST & GDQ-RM & GDQ-GST & GDQ-RM & GDQ-GST & GDQ-RM & GDQ-GST & GDQ-RM \\
\hline$f_{1}$ & 439.664 & 439.768 & 440.999 & 441.043 & 441.317 & 441.345 & 364.415 & 364.341 & 365.394 & 365.339 \\
\hline$f_{2}$ & 447.296 & 447.842 & 449.607 & 450.144 & 450.176 & 450.711 & 375.370 & 375.795 & 375.720 & 376.149 \\
\hline$f_{3}$ & 447.296 & 447.842 & 449.607 & 450.144 & 450.176 & 450.711 & 375.370 & 375.795 & 375.720 & 376.149 \\
\hline$f_{4}$ & 461.762 & 461.978 & 463.248 & 463.374 & 463.637 & 463.779 & 386.898 & 387.106 & 387.431 & 387.621 \\
\hline$f_{5}$ & 461.762 & 462.406 & 464.276 & 464.916 & 464.911 & 465.549 & 389.309 & 389.840 & 389.477 & 390.008 \\
\hline$f_{6}$ & 461.907 & 462.406 & 464.276 & 464.916 & 464.911 & 465.549 & 389.309 & 389.840 & 389.477 & 390.008 \\
\hline$f_{7}$ & 487.146 & 487.753 & 489.657 & 490.261 & 490.422 & 491.024 & 411.574 & 412.079 & 411.661 & 412.166 \\
\hline$f_{8}$ & 487.146 & 487.753 & 489.657 & 490.261 & 490.422 & 491.024 & 411.574 & 412.079 & 411.661 & 412.166 \\
\hline$f_{9}$ & 523.557 & 524.045 & 525.718 & 525.638 & 525.902 & 525.823 & 436.735 & 436.676 & 437.571 & 437.510 \\
\hline$f_{10}$ & 523.557 & 524.045 & 525.718 & 525.638 & 525.902 & 525.823 & 436.735 & 436.676 & 437.571 & 437.510 \\
\hline \multicolumn{11}{|c|}{ Foundation properties: $\rho_{F}^{+}=1800 \mathrm{~kg} / \mathrm{m}^{3}, \quad h_{F}^{+}=0.1 \mathrm{~m}, \quad k_{n}^{+}=7.5 \times 10^{7} \mathrm{~N} / \mathrm{m}^{3}, \quad G_{F}^{+}=5 \times 10^{6} \mathrm{~N} / \mathrm{m}, \quad k_{\varphi}^{+}=k_{s}^{+}=3.75 \times 10^{7} \mathrm{~N} / \mathrm{m}^{3}$} \\
\hline \multirow[t]{2}{*}{$\begin{array}{l}\text { Mode } \\
{[\mathrm{Hz}]}\end{array}$} & \multicolumn{2}{|c|}{$\begin{array}{l}k_{n}^{+}=0, k_{\varphi}^{+}=0, \\
k_{s}^{+}=0, G_{F}^{+}=0 \\
\rho_{F}^{+}=0, h_{F}^{+}=0\end{array}$} & \multicolumn{2}{|c|}{$\begin{array}{l}k_{n}^{+} \neq 0, k_{\varphi}^{+}=0, \\
k_{s}^{+}=0, G_{F}^{+}=0, \\
\rho_{F}^{+}=0, h_{F}^{+}=0\end{array}$} & \multicolumn{2}{|c|}{$\begin{array}{l}k_{n}^{+} \neq 0, k_{\varphi}^{+}=0 \\
k_{s}^{+}=0, G_{F}^{+} \neq 0 \\
\rho_{F}^{+}=0, h_{F}^{+}=0\end{array}$} & \multicolumn{2}{|c|}{$\begin{array}{l}k_{n}^{+} \neq 0, k_{\varphi}^{+}=0, \\
k_{s}^{+}=0, G_{F}^{+} \neq 0, \\
\rho_{F}^{+} \neq 0, h_{F}^{+} \neq 0\end{array}$} & \multicolumn{2}{|c|}{$\begin{array}{l}k_{n}^{+} \neq 0, k_{\varphi}^{+} \neq 0, \\
k_{s}^{+} \neq 0, G_{F}^{+} \neq 0, \\
\rho_{F}^{+} \neq 0, h_{F}^{+} \neq 0\end{array}$} \\
\hline & GDQ-GST & GDQ-RM & GDQ-GST & GDQ-RM & GDQ-GST & GDQ-RM & GDQ-GST & GDQ-RM & GDQ-GST & GDQ-RM \\
\hline$f_{1}$ & 439.664 & 439.768 & 445.042 & 444.884 & 446.622 & 446.002 & 367.911 & 367.675 & 373.636 & 373.453 \\
\hline$f_{2}$ & 447.296 & 447.842 & 458.575 & 459.076 & 463.802 & 462.902 & 386.390 & 386.755 & 388.365 & 388.749 \\
\hline$f_{3}$ & 447.296 & 447.842 & 458.575 & 459.076 & 463.802 & 462.902 & 386.390 & 386.755 & 388.365 & 388.749 \\
\hline$f_{4}$ & 461.762 & 461.978 & 469.744 & 470.056 & 475.005 & 474.089 & 397.332 & 397.681 & 399.100 & 399.403 \\
\hline$f_{5}$ & 461.762 & 462.406 & 474.179 & 474.803 & 480.284 & 479.343 & 402.181 & 402.691 & 403.026 & 403.536 \\
\hline$f_{6}$ & 461.907 & 462.406 & 474.179 & 474.803 & 480.284 & 479.343 & 402.181 & 402.691 & 403.026 & 403.536 \\
\hline$f_{7}$ & 487.146 & 487.753 & 499.571 & 500.162 & 506.979 & 506.030 & 425.495 & 425.981 & 425.925 & 426.412 \\
\hline$f_{8}$ & 487.146 & 487.753 & 499.571 & 500.162 & 506.979 & 506.030 & 425.495 & 425.981 & 425.925 & 426.412 \\
\hline$f_{9}$ & 523.557 & 524.045 & 528.406 & 528.339 & 530.434 & 529.901 & 440.816 & 440.787 & 444.754 & 444.712 \\
\hline$f_{10}$ & 523.557 & 524.045 & 528.406 & 528.339 & 530.434 & 529.901 & 440.816 & 440.787 & 444.754 & 444.712 \\
\hline
\end{tabular}

"Control points and weights of the Bézier curve: $\overline{\boldsymbol{x}}_{1}=\left[\begin{array}{lllll}0.8 & 1.3 & 1.5 & 1.4 & 1.2\end{array}\right], \quad \overline{\boldsymbol{x}}_{3}^{\prime}=\left[\begin{array}{lllll}0 & 0.5 & 1 & 1.5 & 2\end{array}\right], \boldsymbol{w}=\left[\begin{array}{lllll}1 & 1 & 1 & 1 & 1\end{array}\right]$, Geometric characteristics: $\vartheta_{0}=360^{\circ}, h=0.1 \mathrm{~m}, \quad R_{b}=0 \mathrm{~m}$, Orthotropic material: $E_{1}=137.9 \mathrm{GPa}, \quad E_{2}=8.96 \mathrm{GPa}, \quad G_{12}=G_{13}=7.1 \mathrm{GPa}, \quad G_{23}=6.21 \mathrm{GPa}, \quad v_{12}=0.3$, $\rho=1450 \mathrm{~kg} / \mathrm{m}^{3}$. 
Table 6. First ten frequencies for a $(0 / 30)$ CCCC panel resting on Winkler-Pasternak elastic foundation.

\begin{tabular}{|c|c|c|c|c|c|c|c|c|c|c|}
\hline \multicolumn{11}{|c|}{ Foundation properties: $\rho_{F}^{-}=1800 \mathrm{~kg} / \mathrm{m}^{3}, h_{F}^{-}=0.1 \mathrm{~m}, k_{n}^{-}=1.5 \times 10^{7} \mathrm{~N} / \mathrm{m}^{3}, G_{F}^{-}=5 \times 10^{5} \mathrm{~N} / \mathrm{m}, k_{\varphi}^{-}=k_{s}^{-}=0.75 \times 10^{7} \mathrm{~N} / \mathrm{m}^{3}$} \\
\hline \multirow[t]{2}{*}{$\begin{array}{l}\text { Mode } \\
{[\mathrm{Hz}]}\end{array}$} & \multicolumn{2}{|c|}{$\begin{array}{l}k_{n}^{-}=0, k_{\varphi}^{-}=0, \\
k_{s}^{-}=0, G_{F}^{-}=0, \\
\rho_{F}^{-}=0, h_{F}^{-}=0\end{array}$} & \multicolumn{2}{|c|}{$\begin{array}{l}k_{n}^{-} \neq 0, k_{\varphi}^{-}=0, \\
k_{s}^{-}=0, G_{F}^{-}=0, \\
\rho_{F}^{-}=0, h_{F}^{-}=0\end{array}$} & \multicolumn{2}{|c|}{$\begin{array}{l}k_{n}^{-} \neq 0, k_{\varphi}^{-}=0, \\
k_{s}^{-}=0, G_{F}^{-} \neq 0, \\
\rho_{F}^{-}=0, h_{F}^{-}=0\end{array}$} & \multicolumn{2}{|c|}{$\begin{array}{l}k_{n}^{-} \neq 0, k_{\varphi}^{-}=0, \\
k_{s}^{-}=0, G_{F}^{-} \neq 0, \\
\rho_{F}^{-} \neq 0, h_{F}^{-} \neq 0\end{array}$} & \multicolumn{2}{|c|}{$\begin{array}{l}k_{n}^{-} \neq 0, k_{\varphi}^{-} \neq 0, \\
k_{s}^{-} \neq 0, G_{F}^{-} \neq 0 \\
\rho_{F}^{-} \neq 0, h_{F}^{-} \neq 0\end{array}$} \\
\hline & GDQ-GST & GDQ-RM & GDQ-GST & GDQ-RM & GDQ-GST & GDQ-RM & GDQ-GST & GDQ-RM & GDQ-GST & GDQ-RM \\
\hline$f_{1}$ & 340.255 & 340.145 & 343.424 & 343.314 & 344.415 & 344.305 & 289.361 & 289.262 & 289.649 & 289.551 \\
\hline$f_{2}$ & 350.272 & 350.142 & 353.307 & 353.178 & 354.343 & 354.214 & 297.893 & 297.779 & 298.185 & 298.072 \\
\hline$f_{3}$ & 383.041 & 382.642 & 385.835 & 385.437 & 386.912 & 386.515 & 325.387 & 325.053 & 325.644 & 325.312 \\
\hline$f_{4}$ & 431.969 & 431.332 & 434.468 & 433.833 & 435.598 & 434.964 & 366.416 & 365.892 & 366.635 & 366.111 \\
\hline$f_{5}$ & 490.418 & 489.664 & 492.658 & 491.905 & 493.849 & 493.096 & 415.329 & 414.719 & 415.507 & 414.898 \\
\hline$f_{6}$ & 550.776 & 550.109 & 552.802 & 552.136 & 554.056 & 553.391 & 465.703 & 465.187 & 465.853 & 465.337 \\
\hline$f_{7}$ & 601.902 & 601.885 & 603.847 & 603.828 & 605.086 & 605.066 & 507.263 & 507.223 & 507.379 & 507.339 \\
\hline$f_{8}$ & 609.556 & 609.133 & 611.415 & 610.992 & 612.731 & 612.312 & 513.997 & 514.047 & 514.112 & 514.162 \\
\hline$f_{9}$ & 609.726 & 609.801 & 611.641 & 611.713 & 612.928 & 612.995 & 514.617 & 514.308 & 514.746 & 514.437 \\
\hline$f_{10}$ & 625.460 & 625.588 & 627.322 & 627.449 & 628.672 & 628.797 & 527.445 & 527.524 & 527.555 & 527.635 \\
\hline \multicolumn{11}{|c|}{ Foundation properties: $\rho_{F}^{-}=1800 \mathrm{~kg} / \mathrm{m}^{3}, h_{F}^{-}=0.1 \mathrm{~m}, k_{n}^{-}=7.5 \times 10^{7} \mathrm{~N} / \mathrm{m}^{3}, G_{F}^{-}=5 \times 10^{6} \mathrm{~N} / \mathrm{m}, k_{\varphi}^{-}=k_{s}^{-}=3.75 \times 10^{7} \mathrm{~N} / \mathrm{m}^{3}$} \\
\hline \multirow[t]{2}{*}{$\begin{array}{c}\text { Mode } \\
{[\mathrm{Hz}]}\end{array}$} & \multicolumn{2}{|c|}{$\begin{array}{l}k_{n}^{-}=0, k_{\varphi}^{-}=0, \\
k_{s}^{-}=0, G_{F}^{-}=0, \\
\rho_{F}^{-}=0, h_{F}^{-}=0\end{array}$} & \multicolumn{2}{|c|}{$\begin{array}{l}k_{n}^{-} \neq 0, k_{\varphi}^{-}=0, \\
k_{s}^{-}=0, G_{F}^{-}=0, \\
\rho_{F}^{-}=0, h_{F}^{-}=0\end{array}$} & \multicolumn{2}{|c|}{$\begin{array}{l}k_{n}^{-} \neq 0, k_{\varphi}^{-}=0, \\
k_{s}^{-}=0, G_{F}^{-} \neq 0, \\
\rho_{F}^{-}=0, h_{F}^{-}=0\end{array}$} & \multicolumn{2}{|c|}{$\begin{array}{l}k_{n}^{-} \neq 0, k_{\varphi}^{-}=0, \\
k_{s}^{-}=0, G_{F}^{-} \neq 0, \\
\rho_{F}^{-} \neq 0, h_{F}^{-} \neq 0\end{array}$} & \multicolumn{2}{|c|}{$\begin{array}{l}k_{n}^{-} \neq 0, k_{\varphi}^{-} \neq 0, \\
k_{s}^{-} \neq 0, G_{F}^{-} \neq 0, \\
\rho_{F}^{-} \neq 0, h_{F}^{-} \neq 0\end{array}$} \\
\hline & GDQ-GST & GDQ-RM & GDQ-GST & GDQ-RM & GDQ-GST & GDQ-RM & GDQ-GST & GDQ-RM & GDQ-GST & GDQ-RM \\
\hline$f_{1}$ & 340.255 & 340.145 & 355.807 & 355.698 & 365.230 & 365.119 & 306.853 & 306.754 & 308.218 & 308.122 \\
\hline$f_{2}$ & 350.272 & 350.142 & 365.186 & 365.055 & 375.044 & 374.915 & 315.303 & 315.190 & 316.692 & 316.583 \\
\hline$f_{3}$ & 383.041 & 382.642 & 396.807 & 396.411 & 407.115 & 406.728 & 342.384 & 342.060 & 343.617 & 343.298 \\
\hline$f_{4}$ & 431.969 & 431.332 & 444.315 & 443.684 & 455.191 & 454.573 & 382.908 & 382.397 & 383.964 & 383.457 \\
\hline$f_{5}$ & 490.418 & 489.664 & 501.513 & 500.763 & 513.035 & 512.296 & 431.474 & 430.876 & 432.341 & 431.748 \\
\hline$f_{6}$ & 550.776 & 550.109 & 560.830 & 560.164 & 573.021 & 572.361 & 481.654 & 481.142 & 482.386 & 481.877 \\
\hline$f_{7}$ & 601.902 & 601.885 & 611.560 & 611.534 & 623.660 & 623.625 & 522.837 & 522.784 & 523.401 & 523.353 \\
\hline$f_{8}$ & 609.556 & 609.133 & 618.789 & 618.365 & 631.583 & 631.223 & 529.787 & 529.822 & 530.350 & 530.390 \\
\hline$f_{9}$ & 609.726 & 609.801 & 619.240 & 619.302 & 631.844 & 631.828 & 530.535 & 530.220 & 531.161 & 530.847 \\
\hline$f_{10}$ & 625.460 & 625.588 & 634.715 & 634.834 & 647.906 & 648.011 & 543.587 & 543.649 & 544.126 & 544.192 \\
\hline
\end{tabular}

${ }^{*}$ Control points and weights of the Bézier curve: $\overline{\boldsymbol{x}}_{1}=\left[\begin{array}{lllll}0 & 0.5 & 1 & 1.5 & 2\end{array}\right], \quad \overline{\boldsymbol{x}}_{3}^{\prime}=\left[\begin{array}{llllll}0.8 & 1.3 & 1.5 & 1.3 & 0.8\end{array}\right], \quad \boldsymbol{w}=\left[\begin{array}{lllll}1 & 1 & 1 & 1 & 1\end{array}\right]$, Geometric characteristics: $\vartheta_{0}=90^{\circ}, h=0.1 \mathrm{~m}, \quad R_{b}=1 \mathrm{~m}$, Orthotropic material: $E_{1}=137.9 \mathrm{GPa}, \quad E_{2}=8.96 \mathrm{GPa}, \quad G_{12}=G_{13}=7.1 \mathrm{GPa}, \quad G_{23}=6.21 \mathrm{GPa}, \quad v_{12}=0.3$, $\rho=1450 \mathrm{~kg} / \mathrm{m}^{3}$. 
Table 7. First ten frequencies for a (30/60) FC shell resting on Winkler-Pasternak elastic foundation.

\begin{tabular}{|c|c|c|c|c|c|c|c|c|c|c|}
\hline \multicolumn{11}{|c|}{ Foundation properties: $\rho_{F}^{-}=1800 \mathrm{~kg} / \mathrm{m}^{3}, h_{F}^{-}=0.1 \mathrm{~m}, k_{n}^{-}=1.5 \times 10^{7} \mathrm{~N} / \mathrm{m}^{3}, G_{F}^{-}=5 \times 10^{5} \mathrm{~N} / \mathrm{m}, k_{\varphi}^{-}=k_{s}^{-}=0.75 \times 10^{7} \mathrm{~N} / \mathrm{m}^{3}$} \\
\hline \multirow[t]{2}{*}{$\begin{array}{c}\text { Mode } \\
{[\mathrm{Hz}]}\end{array}$} & \multicolumn{2}{|c|}{$\begin{array}{l}k_{n}^{-}=0, k_{\varphi}^{-}=0, \\
k_{s}^{-}=0, G_{F}^{-}=0, \\
\rho_{F}^{-}=0, h_{F}^{-}=0\end{array}$} & \multicolumn{2}{|c|}{$\begin{array}{l}k_{n}^{-} \neq 0, k_{\varphi}^{-}=0, \\
k_{s}^{-}=0, G_{F}^{-}=0, \\
\rho_{F}^{-}=0, h_{F}^{-}=0\end{array}$} & \multicolumn{2}{|c|}{$\begin{array}{l}k_{n}^{-} \neq 0, k_{\varphi}^{-}=0, \\
k_{s}^{-}=0, G_{F}^{-} \neq 0, \\
\rho_{F}^{-}=0, h_{F}^{-}=0\end{array}$} & \multicolumn{2}{|c|}{$\begin{array}{l}k_{n}^{-} \neq 0, k_{\varphi}^{-}=0, \\
k_{s}^{-}=0, G_{F}^{-} \neq 0, \\
\rho_{F}^{-} \neq 0, h_{F}^{-} \neq 0\end{array}$} & \multicolumn{2}{|c|}{$\begin{array}{l}k_{n}^{-} \neq 0, k_{\varphi}^{-} \neq 0, \\
k_{s}^{-} \neq 0, G_{F}^{-} \neq 0, \\
\rho_{F}^{-} \neq 0, h_{F}^{-} \neq 0\end{array}$} \\
\hline & GDQ-GST & GDQ-RM & GDQ-GST & GDQ-RM & GDQ-GST & GDQ-RM & GDQ-GST & GDQ-RM & GDQ-GST & GDQ-RM \\
\hline$f_{1}$ & 72.894 & 73.118 & 85.036 & 85.215 & 85.527 & 85.705 & 71.828 & 71.978 & 73.601 & 73.752 \\
\hline$f_{2}$ & 80.378 & 80.658 & 91.421 & 91.653 & 92.019 & 92.246 & 77.392 & 77.581 & 79.033 & 79.225 \\
\hline$f_{3}$ & 80.378 & 80.658 & 91.421 & 91.653 & 92.019 & 92.246 & 77.392 & 77.581 & 79.033 & 79.225 \\
\hline$f_{4}$ & 100.235 & 101.287 & 109.478 & 110.428 & 110.279 & 111.221 & 92.985 & 93.778 & 94.201 & 94.991 \\
\hline$f_{5}$ & 100.235 & 101.287 & 109.478 & 110.428 & 110.279 & 111.221 & 92.985 & 93.778 & 94.201 & 94.991 \\
\hline$f_{6}$ & 120.959 & 122.408 & 129.744 & 131.116 & 130.877 & 132.261 & 110.158 & 111.325 & 110.812 & 111.964 \\
\hline$f_{7}$ & 120.959 & 122.408 & 129.744 & 131.116 & 130.877 & 132.261 & 110.158 & 111.325 & 110.812 & 111.964 \\
\hline$f_{8}$ & 139.742 & 140.410 & 148.060 & 148.701 & 149.746 & 150.406 & 125.669 & 126.227 & 126.043 & 126.595 \\
\hline$f_{9}$ & 139.742 & 140.410 & 148.060 & 148.701 & 149.746 & 150.406 & 125.669 & 126.227 & 126.043 & 126.595 \\
\hline$f_{10}$ & 166.126 & 166.471 & 173.369 & 173.699 & 175.529 & 175.867 & 147.128 & 147.416 & 147.395 & 147.681 \\
\hline \multicolumn{11}{|c|}{ Foundation properties: $\rho_{F}^{-}=1800 \mathrm{~kg} / \mathrm{m}^{3}, h_{F}^{-}=0.1 \mathrm{~m}, k_{n}^{-}=7.5 \times 10^{7} \mathrm{~N} / \mathrm{m}^{3}, G_{F}^{-}=5 \times 10^{6} \mathrm{~N} / \mathrm{m}, k_{\varphi}^{-}=k_{s}^{-}=3.75 \times 10^{7} \mathrm{~N} / \mathrm{m}^{3}$} \\
\hline \multirow[t]{2}{*}{$\begin{array}{c}\text { Mode } \\
{[\mathrm{Hz}]}\end{array}$} & \multicolumn{2}{|c|}{$\begin{array}{l}k_{n}^{-}=0, k_{\varphi}^{-}=0, \\
k_{s}^{-}=0, G_{F}^{-}=0, \\
\rho_{F}^{-}=0, h_{F}^{-}=0\end{array}$} & \multicolumn{2}{|c|}{$\begin{array}{l}k_{n}^{-} \neq 0, k_{\varphi}^{-}=0, \\
k_{s}^{-}=0, G_{F}^{-}=0, \\
\rho_{F}^{-}=0, h_{F}^{-}=0\end{array}$} & \multicolumn{2}{|c|}{$\begin{array}{l}k_{n}^{-} \neq 0, k_{\varphi}^{-}=0, \\
k_{s}^{-}=0, G_{F}^{-} \neq 0, \\
\rho_{F}^{-}=0, h_{F}^{-}=0\end{array}$} & \multicolumn{2}{|c|}{$\begin{array}{l}k_{n}^{-} \neq 0, k_{\varphi}^{-}=0, \\
k_{s}^{-}=0, G_{F}^{-} \neq 0, \\
\rho_{F}^{-} \neq 0, h_{F}^{-} \neq 0\end{array}$} & \multicolumn{2}{|c|}{$\begin{array}{l}k_{n}^{-} \neq 0, k_{\varphi}^{-} \neq 0, \\
k_{s}^{-} \neq 0, G_{F}^{-} \neq 0, \\
\rho_{F}^{-} \neq 0, h_{F}^{-} \neq 0\end{array}$} \\
\hline & GDQ-GST & GDQ-RM & GDQ-GST & GDQ-RM & GDQ-GST & GDQ-RM & GDQ-GST & GDQ-RM & GDQ-GST & GDQ-RM \\
\hline$f_{1}$ & 72.894 & 73.118 & 121.690 & 121.783 & 124.987 & 125.077 & 104.927 & 105.003 & 111.193 & 111.279 \\
\hline$f_{2}$ & 80.378 & 80.658 & 125.891 & 126.017 & 130.041 & 130.134 & 109.337 & 109.414 & 115.358 & 115.454 \\
\hline$f_{3}$ & 80.378 & 80.658 & 125.891 & 126.017 & 130.041 & 130.134 & 109.337 & 109.414 & 115.358 & 115.454 \\
\hline$f_{4}$ & 100.235 & 101.287 & 140.166 & 140.861 & 146.045 & 146.686 & 123.125 & 123.662 & 127.955 & 128.505 \\
\hline$f_{5}$ & 100.235 & 101.287 & 140.166 & 140.861 & 146.045 & 146.686 & 123.125 & 123.662 & 127.955 & 128.505 \\
\hline$f_{6}$ & 120.959 & 122.408 & 159.875 & 161.052 & 168.401 & 169.659 & 141.751 & 142.810 & 144.550 & 145.569 \\
\hline$f_{7}$ & 120.959 & 122.408 & 159.875 & 161.052 & 168.401 & 169.659 & 141.751 & 142.810 & 144.550 & 145.569 \\
\hline$f_{8}$ & 139.742 & 140.410 & 177.411 & 177.983 & 190.552 & 191.285 & 159.855 & 160.471 & 161.472 & 162.059 \\
\hline$f_{9}$ & 139.742 & 140.410 & 177.411 & 177.983 & 190.552 & 191.285 & 159.855 & 160.471 & 161.472 & 162.059 \\
\hline$f_{10}$ & 166.126 & 166.471 & 199.712 & 200.002 & 207.398 & 207.673 & 173.861 & 174.088 & 175.326 & 175.575 \\
\hline
\end{tabular}

${ }^{*}$ Control points and weights of the Bézier curve: $\overline{\boldsymbol{x}}_{1}=\left[\begin{array}{lllll}0 & 0.3 & 1 & 1.5 & 2\end{array}\right], \overline{\boldsymbol{x}}_{3}^{\prime}=\left[\begin{array}{lllllllll}2 & 1.2 & 0.85 & 0.75 & 0.7\end{array}\right], \boldsymbol{w}=\left[\begin{array}{lllllll}1 & 1 & 1 & 1 & 1\end{array}\right]$, Geometric characteristics: $\vartheta_{0}=360^{\circ}, h=0.1 \mathrm{~m}, R_{b}=2 \mathrm{~m}$, Orthotropic material: $E_{1}=137.9 \mathrm{GPa}, E_{2}=8.96 \mathrm{GPa}, G_{12}=G_{13}=7.1 \mathrm{GPa}, \quad G_{23}=6.21 \mathrm{GPa}, \quad v_{12}=0.3$, $\rho=1450 \mathrm{~kg} / \mathrm{m}^{3}$. 

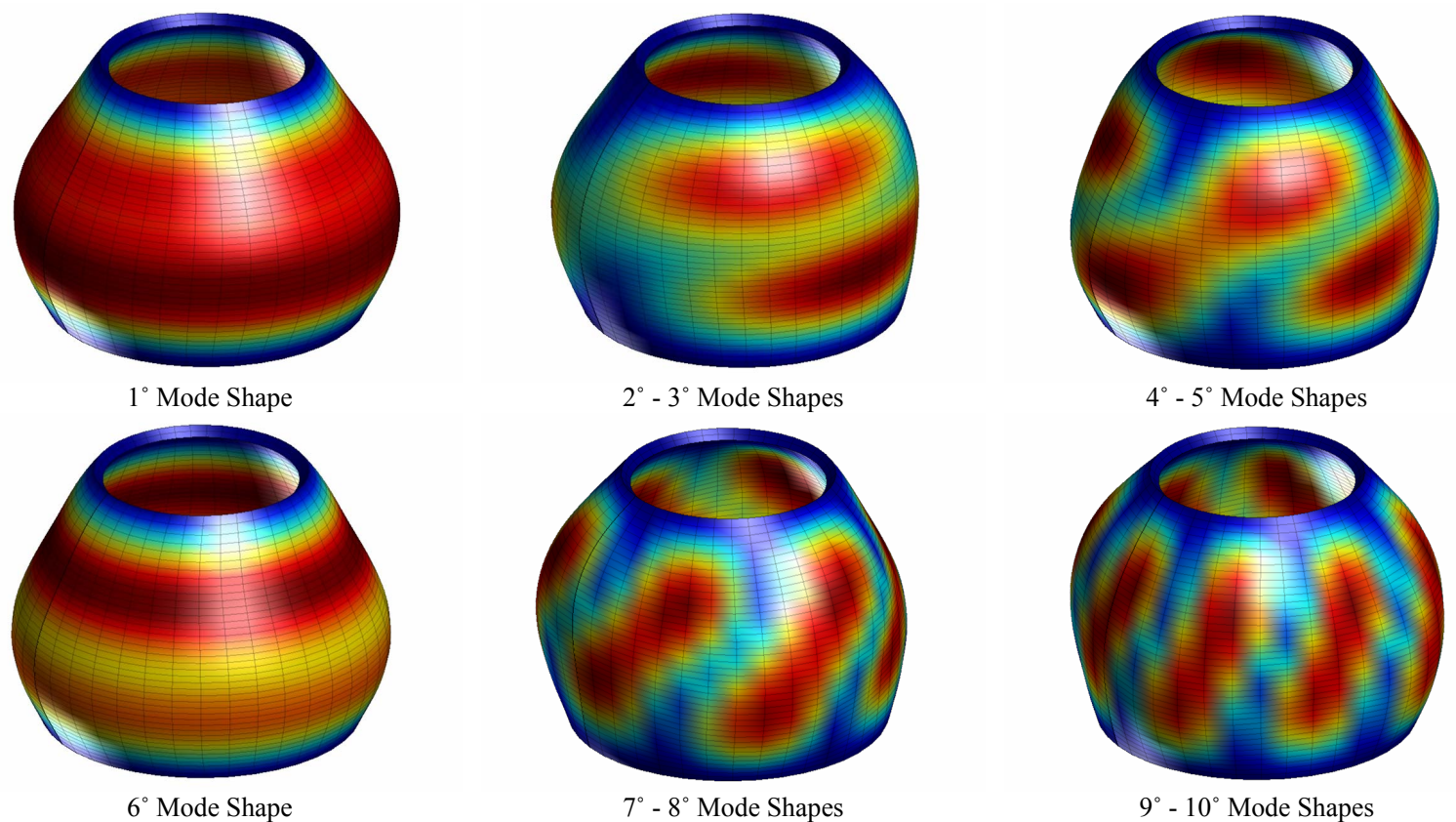

Figure 6. Mode shapes for the CC shell of Table 5.

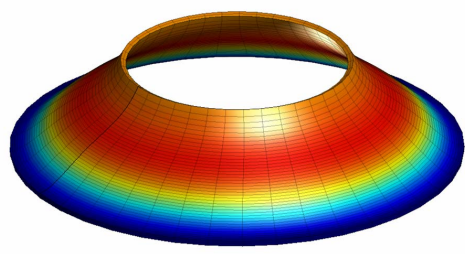

$1^{\circ}$ Mode Shape

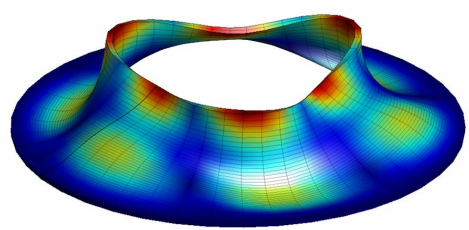

$6^{\circ}-7^{\circ}$ Mode Shapes

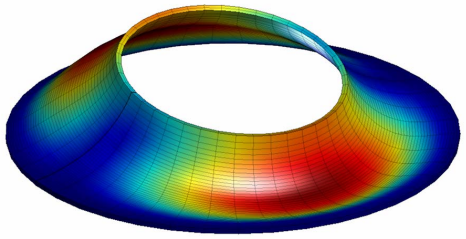

$2^{\circ}-3^{\circ}$ Mode Shapes

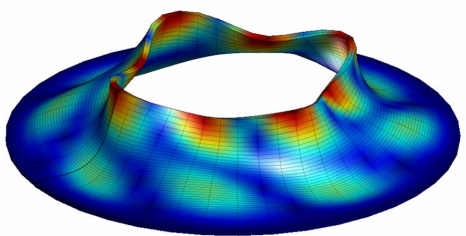

$8^{\circ}-9^{\circ}$ Mode Shapes

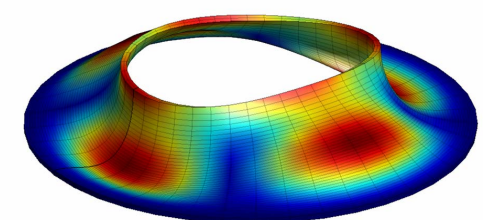

$4^{\circ}-5^{\circ}$ Mode Shapes

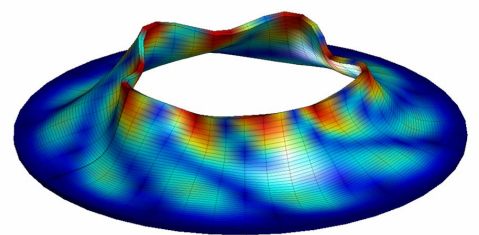

$10^{\circ}-11^{\circ}$ Mode Shape

Figure 7. Mode shapes for the FC shell of Table 7.

Tornabene et al. [69], respectively. In both cases the discrepancy between results obtained is closely zero.

\section{Conclusion}

The static and free vibration analyses of laminated doubly-curved shells and panels of revolution resting on Winkler-Pasternak elastic foundations have been presented using the GDQ method. All the effects of the foundation, except the damping, are separately introduced.
New results are presented in order to investigate the effects of the Winkler modulus, the Pasternak modulus and the inertia of the elastic foundation on the behavior of laminated shells of revolution. Simple Rational Bézier curves are used to mathematically describe the shape of the curved shell. Various lamination schemes with different layers have been considered. A general FSDT shell theory has been obtained considering the curvature effect. The fundamental equilibrium equations have been discretized with the GDQ method giving a standard linear 
problem for the static analysis and a standard linear eigenvalue problem for the dynamic analysis. Numerical solutions have been compared with the ones obtained using commercial programs. The comparisons conducted with FEM codes confirm how the GDQ simple numerical method provides accurate and computationally low cost results for all the structures considered. Furthermore, discretizing and programming procedures are quite easy. The GDQ results show to be precise and reliable. The numerical tests demonstrate and confirm the favorable precision of the Generalized Differential Quadrature Method.

\section{Acknowledgements}

This research was partially supported by the Italian Ministry for University and Scientific, Technological Research MIUR.

\section{REFERENCES}

[1] S. Timoshenko and S. Woinowsky-Krieger, "Theory of Plates and Shells," McGraw-Hill, New York, 1959.

[2] W. Flügge, "Stresses in Shells," Springer-Verlag, Berlin, 1960. doi:10.1007/978-3-662-01028-0

[3] A. L. Gol'denveizer, "Theory of Elastic Thin Shells," Pergamon Press, Oxford, 1961.

[4] V. V. Novozhilov, "Thin Shell Theory," P. Noordhoff, Groningen, 1964.

[5] V. Z. Vlasov, "General Theory of Shells and Its Application in Engineering," NASA-TT-F-99, 1964.

[6] S. A. Ambartusumyan, "Theory of Anisotropic Shells," NASA-TT-F-118, 1964.

[7] H. Kraus, "Thin Elastic Shells," John Wiley \& Sons, Hoboken, 1967.

[8] A. W. Leissa, "Vibration of Plates," NASA-SP-160, 1969.

[9] A. W. Leissa, "Vibration of Shells," NASA-SP-288, 1973.

[10] Š. Markuš, "The Mechanics of Vibrations of Cylindrical Shells," Elsevier, Amsterdam, 1988.

[11] E. Ventsel and T. Krauthammer, "Thin Plates and Shells," Marcel Dekker, New York, 2001. doi:10.1201/9780203908723

[12] W. Soedel, "Vibrations of Shells and Plates," Marcel Dekker, New York, 2004.

[13] E. Reissner, "The Effect of Transverse Shear Deformation on the Bending of Elastic Plates," Journal of Applied Mechanics, Vol. 12, 1945, pp. 66-77.

[14] P. L. Gould, "Finite Element Analysis of Shells of Revolution," Pitman Publishing, New York, 1984.

[15] P. L. Gould, "Analysis of Plates and Shells," PrenticeHall, Upper Saddle River, 1999.

[16] M. S. Qatu, "Accurate Theory for Laminated Composite Deep Thick Shells," International Journal of Solids and Structures, Vol. 36, No. 19, 1999, pp. 2917-2941. doi:10.1016/S0020-76839800134-6
[17] M. S. Qatu, "Vibration of Laminated Shells and Plates," Elsevier, Amsterdam, 2004.

[18] M. H. Toorani and A. A. Lakis, "General Equations of Anisotropic Plates and Shells Including Transverse Shear Deformations, Rotary Inertia and Initial Curvature Effects," Journal of Sound and Vibration, Vol. 237, No. 4, 2000, pp. 561-615. doi:10.1006/jsvi.2000.3073

[19] M. H. Toorani and A. A. Lakis, "Free Vibration of NonUniform Composite Cylindrical Shells," Nuclear Engineering and Design, Vol. 237, No. 17, 2006, pp. 17481758. doi:10.1016/j.nucengdes.2006.01.004

[20] J. N. Reddy, "Mechanics of Laminated Composites Plates and Shells," CRC Press, New York, 2003.

[21] A. Messina, "Free Vibrations of Multilayered Doubly Curved Shells Based on a Mixed Variational Approach and Global Piecewise-Smooth Functions," International Journal of Solids and Structures, Vol. 40, No. 12, 2003, pp. 3069-3088. doi:10.1016/S0020-76830300115-X

[22] C. P. Wu and C. Y. Lee, "Differential Quadrature Solution for the Free Vibration Analysis of Laminated Conical Shells with Variable Stiffness," International Journal of Mechanical Sciences, Vol. 43, No. 8, 2001, pp. 18531869. doi:10.1016/S0020-74030100010-8

[23] A. Ceruti, A. Liverani and G. Caligiana, "Fairing with Neighbourhood LOD Filtering to Upgrade Interactively B-Spline into Class-A Curve," International Journal on Interactive Design and Manufacturing, 2012. http://link.springer.com/article/10.1007\%2Fs12008-012-0 181-9

[24] G. Farin, "Curves and Surfaces for Computer Aided Geometric Design," Academic Press, Waltham, 1990.

[25] L. Piegl and W. Tiller, "The NURBS Book," Springer, Berlin, 1997. doi:10.1007/978-3-642-59223-2

[26] C. Shu, "Differential Quadrature and Its Application in Engineering," Springer, Berlin, 2000. doi:10.1007/978-1-4471-0407-0

[27] D. N. Paliwal, R. K. Pandey and T. Nath, "Free Vibration of Circular Cylindrical Shell on Winkler and Pasternak Foundations," International Journal of Pressure Vessel and Piping, Vol. 69, No. 1, 1996, pp. 79-89. doi:10.1016/0308-01619500010-0

[28] Ö. Civalek, “Geometrically Nonlinear Dynamic Analysis of Doubly Curved Isotropic Shells Resting on Elastin Foundation by a Combination of Harmonic Differential Quadrature-Finite Difference Methods," International Journal of Pressure Vessel and Piping, Vol. 82, No. 6, 2005, pp. 753-761. doi:10.1016/j.ijpvp.2004.12.003

[29] G. B. Golovko, P. Z. Lugovoi and V. F. Meish, "Solution of Axisymmetric Dynamic Problems for Cylindrical Shells on an Elastic Foundation," International Applied Mechanics, Vol. 43, No. 12, 2007, pp. 785-793. doi:10.1007/s10778-008-0006-5

[30] A. H. Sofiyev, "The Buckling of FGM Truncated Conical Shells Subjected to Axial Compressive Load and Resting on Winkler-Pasternak Foundations," International Journal of Pressure Vessel and Piping, Vol. 87, No. 12, 2010, pp. 753-761. doi:10.1016/j.ijpvp.2010.08.012

[31] A. H. Sofiyev, "Buckling Analysis of FGM Circular Shells 
under Combined Loads and Resting on Pasternak Type Elastic Foundations," Mechanics Research Communications, Vol. 37, No. 6, 2010, pp. 539-544. doi:10.1016/j.mechrescom.2010.07.019

[32] C. Bert and M. Malik, "Differential Quadrature Method in Computational Mechanics," Applied Mechanics Reviews, Vol. 49, No. 1, 1996, pp. 1-27. doi:10.1115/1.3101882

[33] K. M. Liew, J. B. Han and Z. M. Xiao, "Differential Quadrature Method for Thick Symmetric Cross-Ply Laminates with First-Order Shear Flexibility," International Journal of Solids and Structures, Vol. 33, No. 18, 1996, pp. 2647-2658. doi:10.1016/0020-76839500174-3

[34] L. Hua and K. Y. Lam, "Frequency Characteristics of a Thin Rotating Cylindrical Shell Using the Generalized Differential Quadrature Method," International Journal of Mechanical Sciences, Vol. 40, No. 5, 1998, pp. 443459. doi:10.1016/S0020-74039700057-X

[35] K. M. Liew and T. M. Teo, "Modeling via Differential Quadrature Method: Three-Dimensional Solutions for Rectangular Plates," Computer Methods in Applied Mechanics and Engineering, Vol. 159, No. 3, 1998, pp. 369381. doi:10.1016/S0045-78259700279-X

[36] T. Y. Ng, H. Li, K. Y. Lam and C. T. Loy, "Parametric Instability of Conical Shells by the Generalized Differential Quadrature Method," International Journal for $\mathrm{Nu}$ merical Methods in Engineering, Vol. 44, No. 6, 1999, pp. 819-837.

doi:10.1002/SICI1097-02071999022844:6<819::AID-N ME528>3.0.CO;2-0

[37] J.-B. Han and K. M. Liew, "Static Analysis of Mindlin Plates: The Differential Quadrature Element Method DQEM," Computer Methods in Applied Mechanics and Engineering, Vol. 177, No. 1-2, 1999, pp. 51-75. doi:10.1016/S0045-78259900371-0

[38] F.-L. Liu and K. M. Liew, "Differential Quadrature Element Method: A New Approach for Free Vibration of Polar Mindlin Plates Having Discontinuities," Computer Methods in Applied Mechanics and Engineering, Vol. 179, No. 3-4, 1999, pp. 407-423. doi:10.1016/S0045-78259900049-3

[39] K. M. Liew and F.-L. Liu, "Differential Quadrature Method for Vibration Analysis of Shear Deformable Annular Sector Plates," Journal of Sound and Vibration, Vol. 230, No. 2, 2000, pp. 335-356. doi:10.1006/jsvi.1999.2623

[40] L. Hua and K. Y. Lam, "Orthotropic Influence on Frequency Characteristics of Rotating Composite Laminated Conical Shell by the Generalized Differential Quadrature Method," International Journal of Solids and Structures, Vol. 38, No. 22, 2001, pp. 3995-4015. doi:10.1016/S0020-76830000272-9

[41] G. Karami and P. Malekzadeh, "A New Differential Quadrature Methodology for Beam Analysis and the Associated Differential Quadrature Element Method," Computer Methods in Applied Mechanics and Engineering, Vol. 191, No. 32, 2002, pp. 3509-3526. doi:10.1016/S0045-78250200289-X

[42] K. M. Liew, T. Y. Ng and J. Z. Zhang, "Differential Qua-
drature-Layerwise Modeling Technique for Three Dimensional Analysis of Cross-Ply Laminated Plates of Various Edge Supports," Computer Methods in Applied Mechanics and Engineering, Vol. 191, No. 35, 2002, pp. 38113832. doi:10.1016/S0045-78250200309-2

[43] T. Y. Wu, Y. Y. Wang and G. R. Liu, "Free Vibration Analysis of Circular Plates Using Generalized Differential Quadrature Rule," Computer Methods in Applied Mechanics and Engineering, Vol. 191, No. 46, 2002, pp. 5365-5380. doi:10.1016/S0045-78250200463-2

[44] T. C. Fung, "Stability and Accuracy of Differential Quadrature Method in Solving Dynamic Problems," Computer Methods in Applied Mechanics and Engineering, Vol. 191, No. 13-14, 2002, pp. 1311-1331. doi:10.1016/S0045-78250100324-3

[45] K. M. Liew and Y. Q. Huang, "Bending and Buckling of Thick Symmetric Rectangular Laminates Using the Moving Least-Squares Differential Quadrature Method," International Journal of Mechanical Sciences, Vol. 45, No. 1, 2003, pp. 95-114. doi:10.1016/S0020-74030300037-7

[46] K. M. Liew, Y. Q. Huang and J. N. Reddy, "Moving Least Squares Differential Quadrature Method and Its Applications to the Analysis of Shear Deformable Plates," International Journal for Numerical Methods in Engineering, Vol. 56, No. 15, 2003, pp. 2332-2351. doi:10.1002/nme.646

[47] K. M. Liew, Y. Q. Huang and J. N. Reddy, "Vibration Analysis of Symmetrically Laminated Plates Based on FSDT Using the Moving Least Squares Differential Quadrature Method," Computer Methods in Applied Mechanics and Engineering, Vol. 192, No. 12, 2003, pp. $2203-$ 2222. doi:10.1016/S0045-78250300238-X

[48] T. Y. Wu, Y. Y. Wang and G. R. Liu, "A Generalized Differential Quadrature Rule for Bending Analyses of Cylindrical Barrel Shells," Computer Methods in Applied Mechanics and Engineering, Vol. 192, No. 13-14, 2003, pp. 1629-1647. doi:10.1016/S0045-78250200650-3

[49] Y. Q. Huang and Q. S. Li, "Bending and Buckling Analysis of Antisymmetric Laminates Using the Moving Least Square Differential Quadrature Method," Computer Methods in Applied Mechanics and Engineering, Vol. 193, No. 33-35, 2004, pp. 3471-3492. doi:10.1016/j.cma.2003.12.039

[50] X. Wang and Y. Wang, "Free Vibration Analyses of Thin Sector Plates by the New Version of Differential Quadrature Method," Computer Methods in Applied Mechanics and Engineering, Vol. 193, No. 36, 2004, pp. 3957-3971. doi:10.1016/j.cma.2004.02.010

[51] P. Malekzadeh, G. Karami and M. Farid, "A Semi-Analytical DQEM for Free Vibration Analysis of Thick Plates with Two Opposite Edges Simply Supported," Computer Methods in Applied Mechanics and Engineering, Vol. 193, No. 45, 2004, pp. 4781-4796. doi:10.1016/j.cma.2004.05.005

[52] Ö. Civalek, "Geometrically Nonlinear Dynamic Analysis of Doubly Curved Isotropic Shells Resting on Elastic Foundation by a Combination of HDQ-FD Methods," International Journal Pressure Vessels and Piping, Vol. 82, No. 6, 2005, pp. 470-479. 
doi:10.1016/j.ijpvp.2004.12.003

[53] E. Viola and F. Tornabene, "Vibration Analysis of Damaged Circular Arches with Varying Cross-Section," Structural Integrity \& Durability, Vol. 1, 2005, pp. 155-169. doi:10.3970/sdhm.2005.001.155

[54] Z. Zong, K. Y. Lam and Y. Y. Zhang, "A Multidomain Differential Quadrature Approach to Plane Elastic Problems with Material Discontinuity," Mathematical and Computer Modelling, Vol. 41, No. 4-5, 2005, pp. 539-553. doi:10.1016/i.mcm.2003.11.009

[55] E. Viola and F. Tornabene, "Vibration Analysis of Conical Shell Structures Using GDQ Method," Far East Journal of Applied Mathematics, Vol. 25, No. 1, 2006, pp. 2339. http://www.pphmj.com/abstract/2166.htm

[56] Ö. Civalek, "Linear Vibration Analysis of Isotropic Conical Shells by Discrete Singular Convolution DSC," International Journal of Structural Engineering and Mechanics, Vol. 25, No. 1, 2007, pp. 127-130.

[57] F. Tornabene, "Modellazione e Soluzione di Strutture a Guscio in Materiale Anisotropo Modelling and Solution of Shell Structures made of Anisotropic Materials," Ph.D. Thesis, DISTART Department, University of Bologna, Bologna, 2007.

[58] F. Tornabene and E. Viola, "Vibration Analysis of Spherical Structural Elements Using the GDQ Method," Computers \& Mathematics with Applications, Vol. 53, No. 10, 2007, pp. 1538-1560. doi:10.1016/j.camwa.2006.03.039

[59] E. Viola, M. Dilena and F. Tornabene, "Analytical and Numerical Results for Vibration Analysis of Multi-Stepped and Multi-Damaged Circular Arches," Journal of Sound and Vibration, Vol. 299, No. 1-2, 2007, pp. 143163. doi:10.1016/j.jsv.2006.07.001

[60] X. Wang, "Nonlinear Stability Analysis of Thin Doubly Curved Orthotropic Shallow Shells by the Differential Quadrature Method," Computer Methods in Applied Mechanics and Engineering, Vol. 196, No. 17-20, 2007, pp. 2242-2251. doi:10.1016/j.cma.2006.11.009

[61] X. Wang, X. Wang and X. Shi, "Accurate Buckling Loads of Thin Rectangular Plates under Parabolic Edge Compressions by the Differential Quadrature Method," International Journal of Mechanical Sciences, Vol. 49, No. 4, 2007, pp. 447-453. doi:10.1016/j.ijmecsci.2006.09.004

[62] A. Marzani, F. Tornabene and E. Viola, "Nonconservative Stability Problems via Generalized Differential Quadrature Method," Journal of Sound and Vibration, Vol. 315, No. 1-2, 2008, pp. 176-196. doi:10.1016/j.jsv.2008.01.056

[63] F. Tornabene and E. Viola, "2-D Solution for Free Vibrations of Parabolic Shells Using Generalized Differential Quadrature Method," European Journal of MechanicsA/Solids, Vol. 27, No. 6, 2008, pp. 1001-1025. doi:10.1016/j.euromechsol.2007.12.007

[64] A. Alibeigloo and R. Modoliat, "Static Analysis of CrossPly Laminated Plates with Integrated Surface Piezoelectric Layers Using Differential Quadrature," Composite Structures, Vol. 88, No. 3, 2009, pp. 342-353. doi:10.1016/j.compstruct.2008.04.018
[65] F. Tornabene, "Vibration Analysis of Functionally Graded Conical, Cylindrical and Annular Shell Structures with a Four-Parameter Power-Law Distribution," Computer Methods in Applied Mechanics and Engineering, Vol. 198, No. 37-40, 2009, pp. 2911-2935. doi:10.1016/i.cma.2009.04.011

[66] F. Tornabene and E. Viola, "Free Vibrations of Four-Parameter Functionally Graded Parabolic Panels and Shell of Revolution," European Journal of Mechanics-A/Solids, Vol. 28, No. 5, 2009, pp. 991-1013. doi:10.1016/j.euromechsol.2009.04.005

[67] F. Tornabene and E. Viola, "Free Vibration Analysis of Functionally Graded Panels and Shells of Revolution," Meccanica, Vol. 44, No. 3, 2009, pp. 255-281. doi:10.1007/s11012-008-9167-x

[68] F. Tornabene, E. Viola and D. J. Inman, "2-D Differential Quadrature Solution for Vibration Analysis of Functionally Graded Conical, Cylindrical and Annular Shell Structures," Journal of Sound and Vibration, Vol. 328, No. 3, 2009, pp. 259-290. doi:10.1016/j.jsv.2009.07.031

[69] E. Viola and F. Tornabene, "Free Vibrations of Three Parameter Functionally Graded Parabolic Panels of Revolution," Mechanics Research Communications, Vol. 36, No. 5, 2009, pp. 587-594. doi:10.1016/j.mechrescom.2009.02.001

[70] L. Yang and S. Zhifei, "Free Vibration of a Functionally Graded Piezoelectric Beam via State-Space Based Differential Quadrature," Composite Structures, Vol. 87, No. 3, 2009, pp. 257-264. doi:10.1016/j.compstruct.2008.01.012

[71] F. Tornabene, A. Marzani, E. Viola and I. Elishakoff, "Critical Flow Speeds of Pipes Conveying Fluid by the Generalized Differential Quadrature Method," Advances in Theoretical and Applied Mechanics, Vol. 3, No. 3, 2010, pp. 121-138.

http://m-hikari.com/atam/atam2010/atam1-4-2010/

[72] A. Alibeigloo and V. Nouri, "Static Analysis of Functionally Graded Cylindrical Shell with Piezoelectric Layers Using Differential Quadrature Method," Composite Structures, Vol. 92, No. 8, 2010, pp. 1775-1785. doi:10.1016/j.compstruct.2010.02.004

[73] A. Andakhshideh, S. Maleki and M. M. Aghdam, "NonLinear Bending Analysis of Laminated Sector Plates Using Generalized Differential Quadrature," Composite Structures, Vol. 92, No. 9, 2010, pp. 2258-2264. doi:10.1016/j.compstruct.2009.08.007

[74] P. Malekzadeh and A. Alibeygi Beni, "Free Vibration of Functionally Graded Arbitrary Straight-Sided Quadrilateral Plates in Thermal Environment," Composite Structures, Vol. 92, No. 11, 2010, pp. 2758-2767. doi:10.1016/j.compstruct.2010.04.011

[75] O. Sepahi, M. R. Forouzan and P. Malekzadeh, "Large Deflection Analysis of Thermo-Mechanical Loaded Annular FGM Plates on Nonlinear Elastic Foundation via DQM," Composite Structures, Vol. 92, No. 10, 2010, pp. 2369-2378. doi:10.1016/j.compstruct.2010.03.011

[76] M. H. Yas and B. Sobhani Aragh, "Three-Dimensional Analysis for Thermoelastic Response of Functionally 
Graded Fiber Reinforced Cylindrical Panel," Composite Structures, Vol. 92, No. 10, 2010, pp. 2391-2399. doi:10.1016/j.compstruct.2010.03.008

[77] F. Tornabene, "Free Vibrations of Laminated Composite Doubly-Curved Shells and Panels of Revolution via the GDQ Method," Computer Methods in Applied Mechanics and Engineering, Vol. 200, No. 9, 2011, pp. 931-952. doi:10.1016/j.cma.2010.11.017

[78] F. Tornabene, "2-D GDQ Solution for Free Vibrations of Anisotropic Doubly-Curved Shells and Panels of Revolution," Composite Structures, Vol. 93, No. 7, 2011, pp. 1854-1876. doi:10.1016/j.compstruct.2011.02.006

[79] F. Tornabene, "Free Vibrations of Anisotropic DoublyCurved Shells and Panels of Revolution with a Free-Form Meridian Resting on Winkler-Pasternak Elastic Foundations," Composite Structures, Vol. 94, No. 1, 2011, pp. 186-206. doi:10.1016/j.compstruct.2011.07.002

[80] F. Tornabene, A. Liverani and G. Caligiana, "FGM and Laminated Doubly Curved Shells and Panels of Revolution with a Free-Form Meridian: A 2-D GDQ Solution for Free Vibrations," International Journal of Mechanical Sciences, Vol. 53, No. 6, 2011, pp. 446-470. doi:10.1016/j.ijmecsci.2011.03.007

[81] F. Tornabene, A. Liverani and G. Caligiana, "Laminated Composite Rectangular and Annular Plates: A GDQ Solution for Static Analysis with a Posteriori Shear and Normal Stress Recovery," Composites Part B: Engineering, Vol. 43, No. 4, 2012, pp. 1847-1872. doi:10.1016/j.compositesb.2012.01.065

[82] F. Tornabene, A. Liverani and G. Caligiana, "General Anisotropic Doubly-Curved Shell Theory: A Differential Quadrature Solution for Free Vibrations of Shells and Panels of Revolution with a Free-Form Meridian," Journal of Sound \& Vibration, Vol. 331, No. 22, 2012, pp. 4848-4869. doi:10.1016/j.jsv.2012.05.036

[83] F. Tornabene, "Meccanica delle Strutture a Guscio in Materiale Composito. Il Metodo Generalizzato di Quadratura Differenziale,” Esculapio, Bologna, 2012.

[84] E. Viola, F. Tornabene and N. Fantuzzi, "General HigherOrder Shear Deformation Theories for the Free Vibration Analysis of Completely Doubly-Curved Laminated Shells and Panels," Composite Structures, Vol. 95, No. 1, 2013, pp. 639-666. doi:10.1016/j.compstruct.2012.08.005

[85] F. Tornabene and E. Viola, "Static Analysis of Functionally Graded Doubly-Curved Shells and Panels of Revolution," Meccanica, 2012. doi:10.1007/s11012-012-9643-1

[86] Sh. Hosseini-Hashemi, M. Fadaee and M. Es'haghi, "A Novel Approach for In-Plane/Out-of-Plane Frequency Analysis of Functionally Graded Circular/Annular Plates," International Journal of Mechanical Sciences, Vol. 52, No. 8, 2010, pp. 1025-1035. doi:10.1016/i.ijmecsci.2010.04.009

[87] X. Zhao and K. M. Liew, "Free Vibration Analysis of Functionally Graded Conical Shell Panels by a Meshless Method," Composite Structures, Vol. 93, No. 2, 2011, pp. 649-664. doi:10.1016/j.compstruct.2010.08.014 


\section{Appendix}

The engineering elastic stiffnesses $\bar{A}_{i j m}^{(q)}$ which depend on curvatures for a doubly-curved shell of revolution are defined as it follows:

$$
\begin{aligned}
& \bar{A}_{i j \varphi}^{(q)}=\sum_{k=1}^{l} \int_{\zeta_{k}}^{\zeta_{k+1}} \bar{Q}_{i j}^{(k)} \zeta^{q} \frac{1+\zeta / R_{s}}{1+\zeta / R_{\varphi}} \mathrm{d} \zeta \\
& \bar{A}_{i j s}^{(q)}=\sum_{k=1}^{l} \int_{\zeta_{k}}^{\zeta_{k+1}} \bar{Q}_{i j}^{(k)} \zeta^{q} \frac{1+\zeta / R_{\varphi}}{1+\zeta / R_{s}} \mathrm{~d} \zeta
\end{aligned}
$$

Using the Taylor geometric series expansion [7,16-18,
85], it is possible to introduce the following approximations:

$$
\begin{aligned}
& \frac{1+\zeta / R_{s}}{1+\zeta / R_{\varphi}} \cong 1+\left(\frac{1}{R_{s}}-\frac{1}{R_{\varphi}}\right) \sum_{p=1}^{r}(-1)^{p+1} \frac{\zeta^{p}}{R_{\varphi}^{p-1}}+o\left(\zeta^{r}\right) \\
& \frac{1+\zeta / R_{s}}{1+\zeta / R_{\varphi}} \cong 1+\left(\frac{1}{R_{s}}-\frac{1}{R_{\varphi}}\right) \sum_{p=1}^{r}(-1)^{p+2} \frac{\zeta^{p}}{R_{s}^{p-1}}+o\left(\zeta^{r}\right)
\end{aligned}
$$

where $r$ is the maximum order of the series expansions. By neglecting the series terms (45) with order higher than $r$ and by introducing the relations (45) into the integrals (44), it is obtained:

$$
\begin{aligned}
& \bar{A}_{i j \varphi}^{(q)} \cong \sum_{k=1}^{l} \int_{\zeta_{k}}^{\zeta_{k+1}} \bar{Q}_{i j}^{(k)} \zeta^{q}\left(1+\left(\frac{1}{R_{s}}-\frac{1}{R_{\varphi}}\right) \sum_{p=1}^{r}(-1)^{p+1} \frac{\zeta^{p}}{R_{\varphi}^{p-1}}\right) \mathrm{d} \zeta \\
& \bar{A}_{i j s}^{(q)} \cong \sum_{k=1}^{l} \int_{\zeta_{k}}^{\zeta_{k+1}} \bar{Q}_{i j}^{(k)} \zeta^{q}\left(1+\left(\frac{1}{R_{s}}-\frac{1}{R_{\varphi}}\right) \sum_{p=1}^{r}(-1)^{p+2} \frac{\zeta^{p}}{R_{s}^{p-1}}\right) \mathrm{d} \zeta
\end{aligned}
$$

Thus, the engineering elastic stiffnesses $\bar{A}_{i j m}^{(q)}$ can be evaluated in the following manner:

$$
\begin{aligned}
& \bar{A}_{i j \varphi}^{(q)} \cong \sum_{k=1}^{l}\left(\int_{\zeta_{k}}^{\zeta_{k+1}} \bar{Q}_{i j}^{(k)} \zeta^{q} \mathrm{~d} \zeta+\left(\frac{1}{R_{s}}-\frac{1}{R_{\varphi}}\right) \sum_{p=1}^{r} \frac{(-1)^{p+1}}{R_{\varphi}^{p-1}} \int_{\zeta_{k}}^{\zeta_{k+1}} \bar{Q}_{i j}^{(k)} \zeta^{q+p} \mathrm{~d} \zeta\right) \\
& \bar{A}_{i j s}^{(q)} \cong \sum_{k=1}^{l}\left(\int_{\zeta_{k}}^{\zeta_{k+1}} \bar{Q}_{i j}^{(k)} \zeta^{q} \mathrm{~d} \zeta+\left(\frac{1}{R_{s}}-\frac{1}{R_{\varphi}}\right) \sum_{p=1}^{r} \frac{(-1)^{p+2}}{R_{s}^{p-1}} \int_{\zeta_{k}}^{\zeta_{k+1}} \bar{Q}_{i j}^{(k)} \zeta^{q+p} \mathrm{~d} \zeta\right)
\end{aligned}
$$

The same results can be exactly obtained using the exlogarithmic function using a Taylor series expansion. Furpressions proposed by Qatu [16], by approximating the

$$
\bar{A}_{i j \varphi}^{(q)} \cong A_{i j}^{(q)}+\left(\frac{1}{R_{s}}-\frac{1}{R_{\varphi}}\right) \sum_{p=1}^{r}(-1)^{p+1} \frac{A_{i j}^{(q+p)}}{R_{\varphi}^{p-1}}, \bar{A}_{i j s}^{(q)} \cong A_{i j}^{(q)}+\left(\frac{1}{R_{s}}-\frac{1}{R_{\varphi}}\right) \sum_{p=1}^{r}(-1)^{p+2} \frac{A_{i j}^{(q+p)}}{R_{s}^{p-1}}
$$

where:

$$
A_{i j}^{(n)}=\sum_{k=1}^{l} \int_{\zeta_{k}}^{\zeta_{k+1}} \bar{Q}_{i j}^{(k)} \zeta^{n} \mathrm{~d} \zeta
$$

Finally, the exact integration of the expressions (47) assume the following aspect:

$$
\begin{aligned}
& \bar{A}_{i j \rho}^{(q)} \cong \sum_{k=1}^{l} \bar{Q}_{i j}^{(k)}\left(\frac{\zeta_{k+1}^{q+1}-\zeta_{k}^{q+1}}{q+1}+\left(\frac{1}{R_{s}}-\frac{1}{R_{\varphi}}\right) \sum_{p=1}^{r} \frac{(-1)^{p+1}}{R_{\varphi}^{p-1}} \frac{\zeta_{k+1}^{q+p+1}-\zeta_{k}^{q+p+1}}{q+p+1}\right) \\
& \bar{A}_{i j s}^{(q)} \cong \sum_{k=1}^{l} \bar{Q}_{i j}^{(k)}\left(\frac{\zeta_{k+1}^{q+1}-\zeta_{k}^{q+1}}{q+1}+\left(\frac{1}{R_{s}}-\frac{1}{R_{\varphi}}\right) \sum_{p=1}^{r} \frac{(-1)^{p+2}}{R_{s}^{p-1}} \frac{\zeta_{k+1}^{q+p+1}-\zeta_{k}^{q+p+1}}{q+p+1}\right)
\end{aligned}
$$

whenever the reduced elastic constants $\bar{Q}_{i j}^{(k)}$ do not depend on $\zeta$ co-ordinate. The engineering elastic stiffness $\bar{A}_{i j m}^{(q)}$ definitions proposed in the present work rep- resent a generalization of the ones proposed in [7,16-18, $77-79,85]$. 